\title{
THESIS
}

\section{ASSOCIATIONS BETWEEN GAIT COORDINATION, VARIABILITY AND MOTOR CORTEX INHIBITION IN YOUNG AND OLDER ADULTS}

\author{
Submitted by \\ Clayton W. Swanson \\ Department of Health \& Exercise Science \\ In partial fulfillment of the requirements \\ For the Degree of Master of Science \\ Colorado State University \\ Fort Collins, Colorado
}

Summer 2018

Master's Committee:

Advisor: Brett W. Fling

Neha Lodha

Agnieszka Burzynska 
Copyright by Clayton W. Swanson 2018

All Rights Reserved 


\begin{abstract}
ASSOCIATIONS BETWEEN GAIT COORDINATION, VARIABILITY AND MOTOR CORTEX INHIBITION IN YOUNG AND OLDER ADULTS
\end{abstract}

Interlimb coordination and mobility (postural sway and turning) diminish with age, posing a risk for gait-related injuries. Further, levels of inhibition within the motor cortex are significantly associated with coordination of the upper extremities in healthy aging, however, it is unknown if this same association exists for lower extremity control. To investigate the relationship between gait coordination and cortical inhibition we measured gait coordination via the phase coordination index and motor cortex inhibition via the cortical silent period in 14 young and 15 older adults. Gait coordination was reduced in older adults across a variety of walking conditions, as was cortical inhibition, solely in the non-dominant motor cortex. Furthermore, young adults were better able to maintain lower extremity coordination and variability with reduced cortical inhibition, whereas older adults with increased cortical inhibition demonstrated better walking performance. These findings suggest a fundamental shift in the relationship between motor cortex inhibition and lower extremity control with age, similar to previous work demonstrating an age-related difference in the association between motor cortex inhibition with bimanual control. 


\section{ACKNOWLEDGEMENTS}

I would like to start by thanking all of the participants who volunteered for this study. They deserve special gratitude for their dedicated time, commitment, and continued trust in our labs research. I am extremely grateful to my mentor Dr. Brett Fling for trusting in me and allowing me to come with him to Colorado State University to continue my education. His scientific knowledge, prowess, understanding of people and genuine ability for mentoring have shaped my graduate career immensely. I cannot thank him enough for his patience, motivation, reassurance, and generosity in completing my degree to the best of my ability.

My graduate life would not be the same without the incredible professors, faculty, and students who all played a role in my studies. Additionally, my family who has been pivotal for my continued interest in education. Both of my parents who have been insightful in maintaining an applied scope to my research. My sisters who have been unconditionally supportive in my education journey and pursuits. Of course, my wife, Timothea who has continued to be a true companion through my graduate education. Her partnership has been unwavering through the long hours and weekends spent completing this degree. Thank you to all who have been supportive, this would not have as much fun without all of the wonderful people who have helped me along the way. 


\section{TABLE OF CONTENTS}

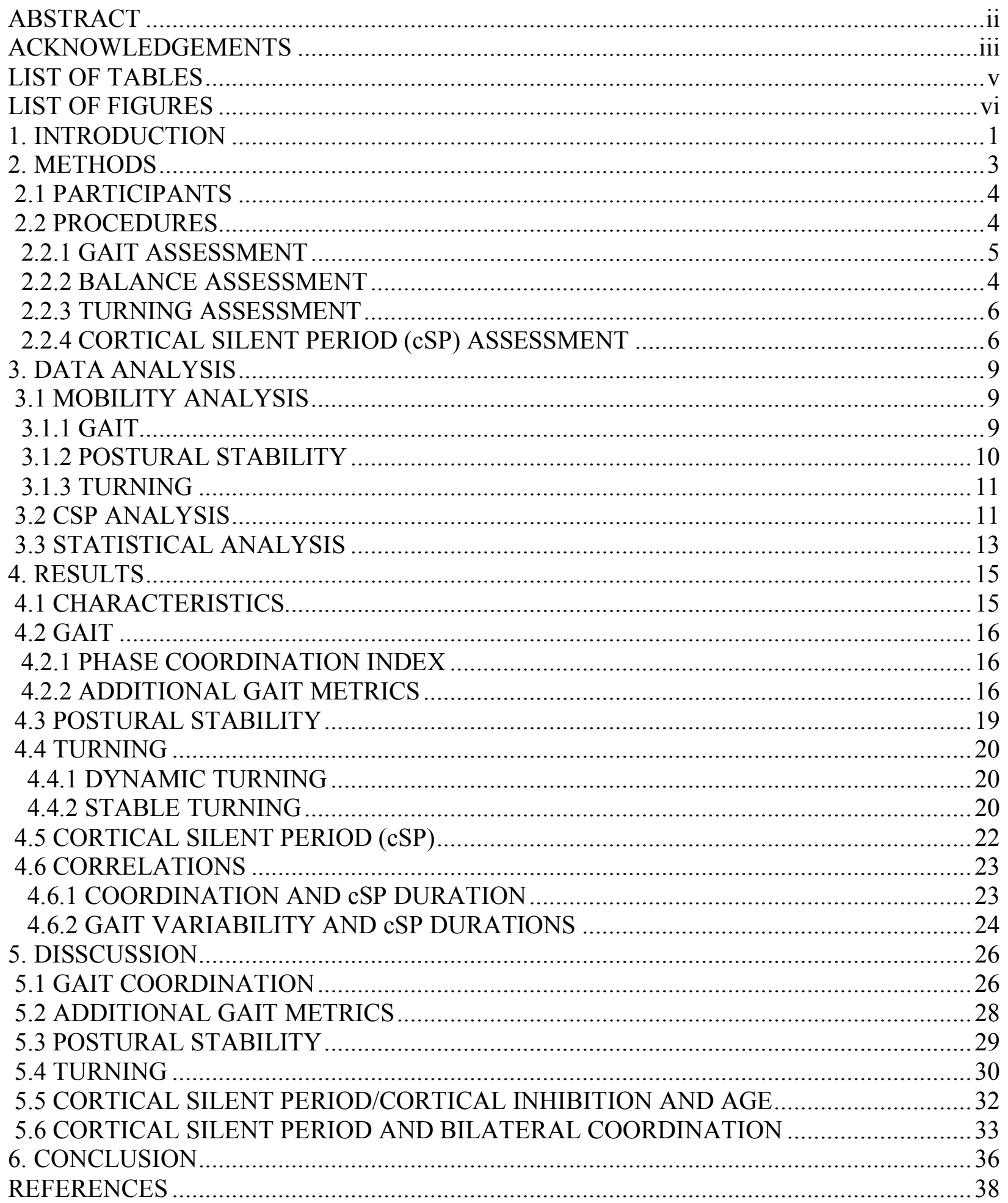




\section{LIST OF TABLES}

TABLE 1 - PARTICIPANT CHARACTERISTICS ........................................................ 15

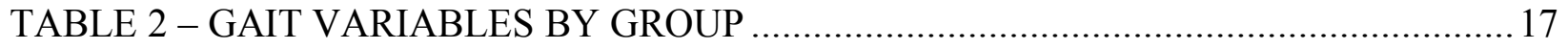

TABLE 3 - GAIT VARIABLES BY CONDITION ........................................................ 18

TABLE 4 - POSTURAL STABILITY VARIABLES BY GROUP...................................... 19

TABLE 5 - DYNAMIC AND STABLE TURN VARIABLES BY GROUP ..........................21

TABLE 6 - DYNAMIC TURN VARIABLES BY CONDITION .......................................21 


\section{LIST OF FIGURES}

FIGURE 1 - EMG TRACE OF CORTICAL SILENT PERIOD ........................................... 12

FIGURE 2 - CORTICAL SILENT PERIOD BY GROUP AND HEMISPHERE.....................22

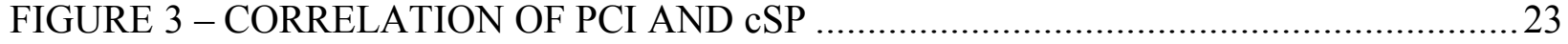

FIGURE 4 - FISHER R - TO - Z TRANSFORMATIONS ..............................................2 25 


\section{INTRODUCTION}

The ability to perform movements that involve interlimb coordination is fundamental to many tasks of daily living, including the manipulation of objects with our hands, standing up from a chair, walking, and climbing stairs (Fujiyama, Hinder, Schmidt, Garry, \& Summers, 2012; James, Leveille, Hausdorff, Barton, et al., 2017; Penninx et al., 2000). Moreover, increasing age is associated with a lack of coordination, causing deficits in our ability to coordinate two limbs together to complete specific tasks. An ever-evolving body of literature indicates that walking incorporates a specific set of movements that involve both spatial and temporal coordination of the lower extremities, and that this interlimb coordination is vital to our ability to control both legs in time and space to ambulate effectively and safely (Brach, Berthold, Craik, VanSwearingen, \& Newman, 2001; Brach, Studenski, Perera, VanSwearingen, \& Newman, 2007; Jeffrey M. Hausdorff, Rios, \& Edelberg, 2001; James, Leveille, Hausdorff, Barton, et al., 2017; James et al., 2016).

Although traditional spatiotemporal metrics of gait are useful in assessing mobility, coordination and variability measures appear to distinguish gait limitations with more specificity (Brach et al., 2001; Brach et al., 2007; Dingwell, Salinas, \& Cusumano, 2017; James et al., 2016; Kang \& Dingwell). Gait coordination is the ability to appropriately time left-right stepping patters within the construct of a stride during walking, commonly quantified using the phase coordination index (PCI) (James, Leveille, Hausdorff, Barton, et al., 2017; Kribus-Shmiel, Zeilig, Sokolovski, \& Plotnik, 2018; M. Plotnik, Giladi, \& Hausdorff, 2007). Gait variability is typically defined as the kinetic stride-to-stride fluctuations of multiple gait cycles over a period of time and distance (Lord, Howe, Greenland, Simpson, \& Rochester, 2011). Both measures are 
sensitive enough to discern between older individuals with and without mobility and cognitive impairments or as a predictor of future fall risk (Jeffrey M Hausdorff, Cudkowicz, Firtion, Wei, \& Goldberger, 1998; James, Leveille, Hausdorff, Barton, et al., 2017; Lord et al., 2011;

Verghese, Holtzer, Lipton, \& Wang, 2009). While coordination has not been studied to the same degree as variability, coordination lacks a strong correlation to variability, suggesting PCI depicts a distinct feature of bilateral gait control and is increased (i.e. poorer coordination) with age (Peterson, Plotnik, Hausdorff, \& Earhart, 2012; M. Plotnik et al., 2007; Yogev, Plotnik, Peretz, Giladi, \& Hausdorff, 2007). While these studies are descriptive, the neural mechanisms that underlie both typical and atypical interlimb gait coordination remain unclear.

With advancing age, there are multi-faceted declines in motor performance as well as changes in cortical inhibition (Fling, Kwak, Peltier, \& Seidler, 2012; Hermans et al.; Hortobágyi, del Olmo, \& Rothwell, 2006; Oliviero et al., 2006; Papegaaij, Taube, Hogenhout, Baudry, \& Hortobágyi, 2014; Peinemann, Lehner, Conrad, \& Siebner, 2001; Pitcher, Ogston, \& Miles, 2003). Transcranial magnetic stimulation (TMS) offers a non-invasive method of studying the inhibitory capacity of the motor cortex believed to reflect excitation and inhibition of gammaaminobutyric acid (GABA) or GABA-ergic cortical circuits (Bhandari et al., 2016; Cash et al., 2017; Di Lazzaro et al., 2000; Kujirai et al., 1993; Lazzaro et al., 1998; Ziemann, Lönnecker, Steinhoff, \& Paulus, 1996). The cortical silent period (cSP) is a common method of assessing GABA-ergic circuits and the inhibitory properties of corticospinal neurons. The cSP refers to an interruption of a voluntary muscle contraction initiated by a TMS stimuli used to assess the health of the corticospinal tract stemming from a particular region of interest within the motor cortex. While a vast body of literature exists demonstrating the associations between motor cortex inhibition, upper extremity control, and the effects of healthy aging (Fling \& Seidler, 
2012; Fujiyama, Garry, Levin, Swinnen, \& Summers, 2009; Fujiyama et al., 2012; Oliviero et al., 2006), there remains a substantial lack of knowledge regarding how cortical inhibition is associated with lower extremity coordination and gait variability.

The purpose of this study was to evaluate the effects of healthy aging on gait coordination and its associations to motor cortex inhibition. Additionally, postural sway and turning metrics were assessed for differences between groups. Over two days of testing, healthy young (YA) and older adults (OA); 1) wore wireless inertial sensors to collect spatiotemporal measures of gait during three different walking conditions and 2) underwent TMS to assess the $\mathrm{cSP}$ of both the dominant and non-dominant leg regions of the primary motor cortices, respectively. We hypothesized that healthy older individuals would have a significantly increased PCI and a significantly shorter cSP compared to their younger counterparts. Finally, we hypothesized that PCI would be significantly correlated with cSP duration in OA, demonstrating a similar association between motor cortex inhibition and lower extremity coordination to those typically observed with the upper extremities during bimanual movements (Boisgontier \& Swinnen, 2015; Fling \& Seidler, 2012; Fujiyama et al., 2009). 


\section{METHODS}

\subsection{Participants}

Twenty-nine healthy adults participated in the study; 14 young participants ( 6 females; age range, 20-31 years; mean age, $24.4 \pm 3.6$ years) and 15 older participants ( 6 females; age range, $65-83$ years; mean age $72.3 \pm 5.7$ years). All participants were able to ambulate independently with no acute fall history (prior 6 months) and had no diagnosed neuromuscular, neurodegenerative, cognitive, orthopedic, or other comorbidities that would impact their gait or risk of TMS. All subjects were either screened in-person or over the phone, once eligibility was determined participants were scheduled for two separate visits within ten days of each other. In addition, prior to enrollment, all subjects were required to score greater than 27 on the Mini Mental State Exam (MMSE) (Tombaugh \& McIntyre, 1992). This study was performed in accordance with the Declaration of Helsinki and approved by the Colorado State University Institutional Review Board, all participants provided written informed consent prior to participating.

\subsection{Procedures}

Participants had two testing sessions which were separated by greater than 24 hours and less than 10 days. Testing sessions included an instrumented assessment of gait and TMS testing. To complete the instrumented assessment, six wireless inertial sensors were positioned on each foot, around the posterior pelvis at the level of L5, on the sternum, and around each wrist (M. Mancini et al., 2011). All sensors were attached to the body using Velcro and elastic straps. Sensors were fit tight enough to limit unwanted sensor movement without being uncomfortable for the participant. 


\subsubsection{Gait Assessment}

Instrumented assessment of gait was conducted in three conditions: single-task, dual-task (DT), and fast-paced walking. Participants were asked to walk back and fourth down a well-lit hallway of 30 meters in length. The walkway was marked at each end with visible tape to indicated where participants should turn around, tape was used instead of a cone to mimic a natural turn and minimize distraction. In all walking conditions, participants were asked to walk while maintaining a forward gaze and to not communicate with the tester for the duration of the trial. In the single task condition participants were asked to walk at their self-selected natural pace for a total of six minutes. In the DT condition, participants were asked to count aloud backwards by sevens from 345 while concurrently walking at their self-selected pace for two minutes (Springer et al., 2006). Recordings of the DT were collected to evaluate counting performance based on number achieved, errors and accuracy. Two older participants were removed from this analysis based on recording issues. The fast condition consisted of walking as fast as possible for two minutes making sure to have one foot on the ground at all times throughout the gait cycle. This condition was included based on prior research demonstrating increased gait variability changes in older individuals with fast walking speeds (Brach et al., 2007; Kang \& Dingwell, 2007).

\subsubsection{Balance Assessment}

Balance was assessed using the Clinical Test of Sensory Organization and Balance (CTSIB). Objective measures of balance were quantified in addition to the standard clinical score. The CTSIB includes four individual conditions each lasting 30-seconds: eye open on a firm surface, eyes closed on a firm surface, eyes open on a compliant surface, and eyes closed on a compliant surface (F. B. Horak, 1987; Shumway-Cook \& Horak, 1986). While balancing 
during the eyes open trials, participants were asked to look straight ahead at a fixed target of 2.5 meters away. If a participant was not able to maintain their balance for the duration of the trial, they were allowed a second chance. In the cases where they were still unable to maintain their balance or felt unsafe that trial was removed from further data analysis.

\subsubsection{Turning Assessment}

The assessment of turning included both dynamic and stable turns. Dynamic turns were defined as turns occurring during bouts of walking. Stable turns were defined as turns occurring in place, similar to a turn made in a small area such as a kitchen or bathroom. For stable turns participants were asked to conduct one $360^{\circ}$ turn with an immediate turn in the opposite direction after the first $360^{\circ}$ turn. For all participants, the first turn was completed in the clockwise direction and the second turn in the counter clock wise direction. Participants were asked not to spin on their toes or heals ensuring they were conducting a natural stepping pattern. Furthermore, participants were asked to complete the two turns as fast and safely as possible. The turning assessment included three separate trials, prior to each trial their toes were lined up with a reference line on the floor and had a foot template placed between their feet to ensure all participants started with the same stance width. In case of confusion, a demonstration was provided for participants who were unsure of the verbal instructions furthermore, if a trial was completed improperly, additional trials were allowed.

\subsubsection{Cortical Silent Period (cSP) Assessment}

Subjects were seated in an adjustable upright chair with their legs off the ground. Motor evoked potentials (MEPs) were elicited in the vastus medialis oblique (VMO) muscle of each leg using a MagPro X100 stimulator (MagVenture, Farum, Denmark) using a 2 x 95mm angled butterfly coil (120-degree, Cool D-B80). In order to be consistent across participants the scalp 
was mapped using permanent marker. The center of the head was determined by measuring from the nasion to occipital protuberance and from the center of each external auditory canal. Once the center of the scalp was marked, a mark was made laterally to each side at $2.0 \mathrm{~cm}$ and anterior 5.5 cm (Groppa et al., 2012). Once the initial markings were made, a sagittal line was drawn as a reference point for the coil. With participants seated and relaxed, the 'hot spot' for cortical stimulation of the VMO was determined as the location where the stimulus evoked a maximal EMG response from the VMO. The resting motor threshold (RMT) was determined in both hemispheres and defined as the lowest stimulus intensity that evoked a response of at least $50 \mu \mathrm{V}$ on five out of ten trials. Muscle activity was recorded via bipolar electromyography (EMG) electrodes (Ag-AgCl, 8-mm diameter, 20-mm distance between electrodes, MVAP Medical Supplies Inc.) sampled at $1500 \mathrm{~Hz}$ and transmitted to a laboratory computer.

Participants were asked to produce a series of maximal voluntary contractions (MVCs) to determine their maximal force output. Participants' legs (individually) were secured to the chair using a strap around the posterior shank and attached to a force transducer and adjustable bar beneath the participant. The bar height was adjusted for each participant to account for leg length and seated foot width, making sure the force transducer was plumb and parallel to the floor. Each subject conducted between two-five MVC's and was concluded when force production no longer increased across attempts and the two highest values were within $10 \%$ of each other. The same process was replicated for the opposite leg.

The cSP was tested for both hemispheres and the corresponding leg, the order of testing (i.e. right vs left) was randomized across participants. To elicit the cSP, participants were asked to maintain an isometric contraction at $15 \%$ of their MVC. Participants were given visual biofeedback on a screen directly in front of them, which depicted a vertical bar that grew or 
shrunk in accordance to the force being produced. A target line on the screen revealed where the bar should be maintained for the duration of the trial (i.e. $15 \%$ of MVC). Participants were asked to maintain the force as steady as possible during the trial. Each trial was two minutes long, during which time the researcher gave a stimulation at $120 \%$ of the RMT every $7-10$ seconds with a total number of stimulations averaging 12 per hemisphere (24 total) (Fling \& Seidler, 2012). 


\section{DATA ANALYSIS}

Gait, postural stability and turning data were collected using Opal wireless inertial sensors (128Hz), and Mobility Lab software (Version 2) (Opal Sensors, APDM Inc., Portland, OR) was used to automatically stream and export gait metrics (F. Horak, King, \& Mancini, 2015). All gait measures were compared across three walking conditions: single task, DT, and fast. The primary gait measure was PCI with additional measures consisting of: gait speed (m/s), stance (\% gait cycle time (\%GCT)), swing (\%GCT), cadence (steps/min) for left and right legs, double support (\%GCT), foot strike angle (degrees), toe off angle (degrees), single limb support (\%GCT), stride length (m), gait cycle duration (s), and step duration (s).

Balance measures were compared across all four conditions: eyes open firm surface, eyes closed firm surface, eyes open foam surface, eyes closed foam surface. Measures included: 95\%

ellipse sway area $(\mathrm{m} 2 / \mathrm{s} 4)$, jerk (m2/s5), root mean square (RMS) sway $(\mathrm{m} / \mathrm{s} 2)$, range $(\mathrm{m} / \mathrm{s} 2)$, path length $(\mathrm{m} / \mathrm{s} 2)$, and mean velocity $(\mathrm{m} / \mathrm{s})$.

Dynamic turns were compared across all three walking conditions. Dynamic turning metrics included: turn duration (s), turn velocity (degree/s), and number of steps in turn (\#). Stable turning metrics were averaged across all three trials after assessing for significant differences. Stable turning metrics included: turn angle (degree), turn duration (s), turn velocity (degree/s), turn angle accuracy (AU).

\subsection{Mobility analysis}

\subsubsection{Gait}

Interlimb bilateral coordination was determined using PCI. Phase coordination index measures the degree of consistency and accuracy in generating a series of anti-phase left-right 
stepping phases (Kribus-Shmiel et al.; M. Plotnik et al., 2007). The PCI calculation has been described previously (M. Plotnik et al., 2007). Briefly, PCI is the combination and summation of two measures representing the relative timing of contralateral heel strikes thus determining phase, represented as phi $(\varphi)$. Phi is calculated through the normalization of step time with respect to stride time (i.e. a $\varphi=180^{\circ}$ for each step is ideal). Once $\varphi$ is determined $\varphi \mathrm{CoV}$ and $\varphi \mathrm{ABS}$ are summed to give PCI (Equation 1.), with a lower value equating to better phase control and coordination (i.e. $180^{\circ}=$ ideal interlimb coordination).

Equation 1. Formula for the calculation of $\varphi \mathrm{CoV}$ and $\varphi \mathrm{ABS}$ for PCI.

$$
\begin{gathered}
\varphi \operatorname{CoV}=\frac{\operatorname{stdev}(\varphi)}{\operatorname{mean}(\varphi)} \times 100 \\
\varphi A B S=\frac{\operatorname{mean}\left(\left|\varphi-180^{\circ}\right|\right)}{180^{\circ}} \times 100 \\
\operatorname{PCI}(\%)=\varphi \operatorname{CoV}+\varphi \mathrm{ABS}
\end{gathered}
$$

Additional gait outcomes included more traditional means and variability measures. Variability was measured using coefficients of variation (CoV) (SD/Mean) a commonly used measure to quantify the magnitude of variability. Gait metrics were chosen based on previous findings indicating sensitive measurements for instrumented assessment of OA (Maki, 1997; Park, Mancini, Carlson-Kuhta, Nutt, \& Horak, 2016).

\subsubsection{Postural Stability}

Postural stability metrics were collected during the CTSIB using wireless inertial sensors and Mobility Lab Software (Version 2). All postural stability measures were automatically calculated and exported for further analysis. All measures were chosen based on previous literature demonstrating their metric sensitivity for the assessment of postural control within the 
different domains of balance (i.e. jerk, temporal, and frequency measures) (M. Mancini et al., 2012; Martina Mancini et al., 2016; Park et al., 2016).

\subsubsection{Turning}

Dynamic turning was collected during each of the three independent walking conditions using inertial sensors and Mobility Lab Software (Version 2). All dynamic turning metrics were automatically calculated and exported for further analysis.

Stable turning was quantified using inertial sensors and Mobility Lab software (Version 2). However, turn angle accuracy. was assessed by adjusting for the turn degree achieved compared to the turn degree assumed for the specific trial turn angle (e.g. $180^{\circ}$ for dynamic and $360^{\circ}$ for stable) (Equation 2). For instance, if a participant turned $375^{\circ}$ but was asked to turn $360^{\circ}$ the difference of $15^{\circ}$ was calculated as their turn accuracy.

Equation 2. Formula to calculate turn accuracy.

$$
\text { Turn Accuracy }=(\text { Turn Degree Achieved }- \text { Turn Degree Expected })
$$

All turning metrics (turn angle, turn duration, steps in turn, and turn velocity) except for turn accuracy were chosen based on prior literature demonstrating their sensitivity in assessing OA with increased fall risk (Martina Mancini et al., 2016; Park et al., 2016). Turn accuracy is a novel assessment developed in our lab to assess how accurately an individual is capable of turning a pre-designated amount.

\section{2 cSP Analysis}

EMG signals were filtered and rectified offline using AcqKnowledge software (Biopac Inc., Santa Barbara, CA). Filtered and rectified data was then imported into a custom MATLAB (MathWorks, Nantick, MA) script to identify and calculate individual cSPs providing an average cSP duration for each leg following standard approaches (Fisher et al., 2008). Briefly, for a given 
leg, the EMG signal was extracted from $100 \mathrm{~ms}$ prior to each stimulation to $350 \mathrm{~ms}$ post stimulation. Duration of the cSP was quantified by identifying the mean EMG value for the $100 \mathrm{~ms}$ prior to each stimulation. Following stimulation, cSP onset was identified as the point when EMG activity dropped below 1.5 standard deviations of the pre-stimulus mean (Figure 1). Offset of the cSP was defined as the point in time when five consecutive data points were greater than 1.5 standard deviations below the pre-stimulus EMG mean (Fling \& Seidler, 2012; Garvey \& Gilbert).
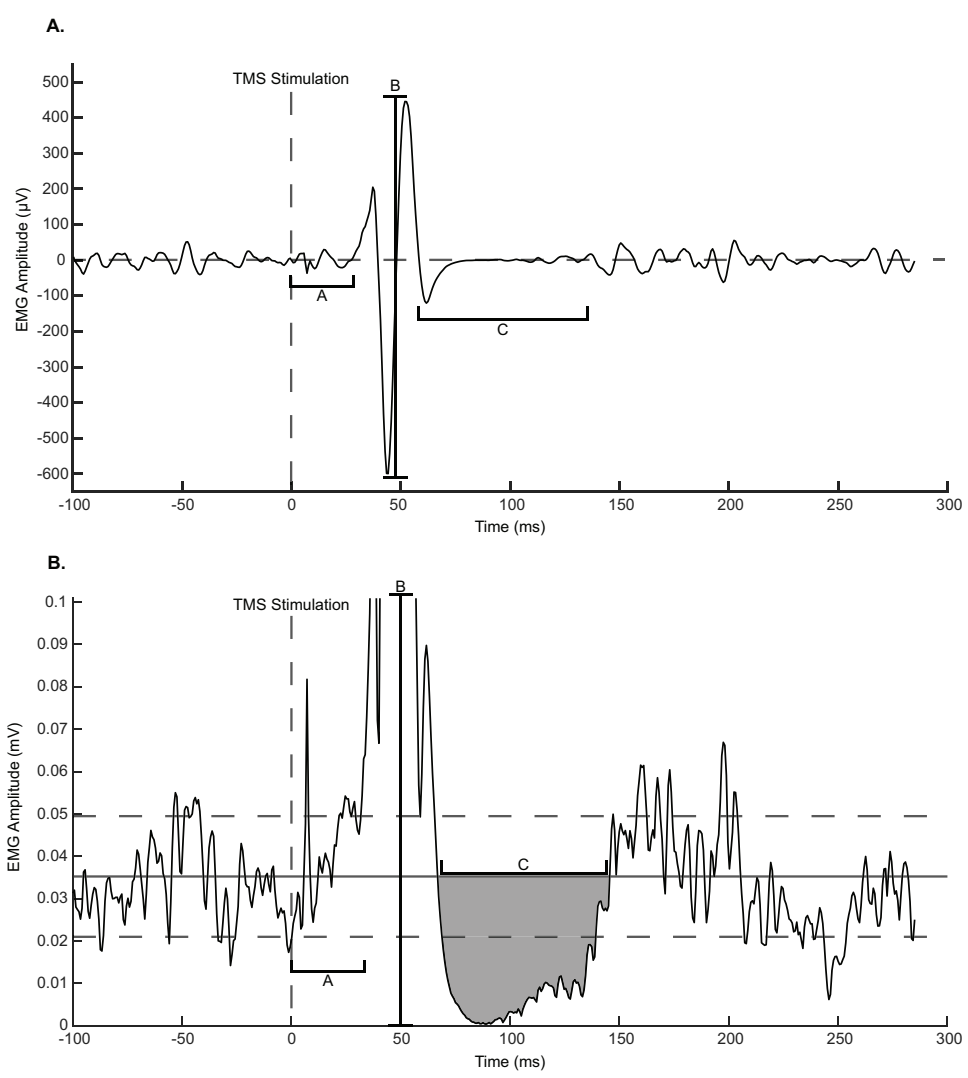

Figure 1. Quantification of TMS and cSP from a representative participant. A) Signifies the representative response from TMS to the left motor cortex after the EMG signal has been filtered. B) EMG signal after filtering and rectification with the grey indicating the silent period. Pre-stimulus mean indicated by a solid line, with the $\pm 1.5 \mathrm{SD}$ of the pre-stimulus mean indicated by the dashed lines. Within both $1 \mathrm{~A}$ and $1 \mathrm{~B}$ we identify the latency period (A), motor evoked potential (B) and silent period (C). 


\subsection{Statistical Analysis}

All statistical analysis was conducted in JMP Pro 13 with an alpha level set at 0.05 . Between-group differences for demographic variables were assessed using independent t-tests. During data processing three participants (two YA, one OA) did not have quantifiable TMS measures, therefore, their data was excluded from further analysis. An exploratory outlier test was completed, outliers were defined as values three interquartile ranges past the 25 th and 75 th percentile tails. This resulted in three PCI, eleven postural stability, and one cSP data point removed from further analysis.

A $2 \times 3$ repeated measure analysis of variance (ANOVA) to assess for group and condition main effects as well as any group by condition interactions was utilized for all gait analysis. Significant main effects were assessed post-hoc using Student's t-test corrected with a Tukey-Kramer HSD, which corrects for the number of independent pair-wise comparisons. A $2 \mathrm{x}$ 4 ANOVA to assess for group and balance condition main effects as well as any group by condition interactions was applied to all postural analysis. As with gait analysis significant main effects were assessed post-hoc using Student's t-test corrected with a Tukey-Kramer HSD. For dynamic stability analyses, a 2 x 3 ANOVA was used to assess turning differences between the three different walking conditions. For stable turning analyses, a 2 x 3 ANOVA was used to assess differences between trials. Subsequently, there were no significant differences between stable turning trials therefore the average of the three trials was used for further analysis, where an independent t-test was utilized to assess group differences. For motor cortex inhibition, 2 x 2 ANOVA was chosen to assess for significant group differences between hemispheric cSP durations. 
Linear regression was used to identify correlations between cSP duration (hemisphere specific) and gait metrics for the three walking conditions. The correlation strength between each cSP duration and the different walking conditions was calculated using Pearson correlation coefficients creating a correlation matrix of the cSP durations and gait metric values. For all significant Pearson correlations, values were further evaluated via a Fischer's r-to-z transformation to identify differences in the strength of correlations between groups and conditions (Zar, 1998). All data are presented as mean \pm standard deviation unless otherwise noted. 


\section{RESULTS}

\subsection{Characteristics}

Characteristics of the study participants are presented in Table 1. Age was significantly different between YA and OA groups (difference in mean age 48.0 years, $\mathrm{t}(27)=26.97, \mathrm{p}<$ 0.001). The comparison between groups indicated YA exercised at a higher intensity on average $(\mathrm{F}(1,27)=4.46, \mathrm{p}=0.044)$, furthermore YA perceived less exertion post testing $(\mathrm{F}(1,27)=8.13$, $\mathrm{p}=0.008)$. No other characteristic metrics were significant.

Table 1. Demographic, anthropometric, activity information, and rate of perceived exertion (RPE) scale. Values are mean (SD) unless otherwise noted; $p$ values refer to the main effect across age groups.

\begin{tabular}{llll}
\hline & $\begin{array}{l}\text { Younger Adults } \\
(\mathrm{n}=14)\end{array}$ & $\begin{array}{l}\text { Older Adults } \\
(\mathrm{n}=15)\end{array}$ & $p$ value \\
\hline Characteristics & $6(35.70)$ & $6(40.00)$ & \\
Age (years) & $24.36(3.56)$ & $72.33(5.68)$ & $<0.001$ \\
Height (cm) & $172.72(8.10)$ & $172.05(11.06)$ & 0.855 \\
Mass (kg) & $69.04(13.77)$ & $79.29(16.17)$ & 0.078 \\
Dominate Leg (n, \% Right) & $11(78.57)$ & $15(100.00)$ & \\
Right Leg Length (cm) & $88.30(5.10)$ & $92.20(5.66)$ & 0.159 \\
Left Leg Length (cm) & $88.04(5.30)$ & $92.16(5.87)$ & 0.058 \\
Activity Frequency (days) & $4.57(1.41)$ & $4.23(1.95)$ & 0.600 \\
Activity Duration (mins) & $65.36(18.76)$ & $57.00(33.16)$ & 0.415 \\
Average Activity Intensity & $14.36(1.74)$ & $12.07(3.69)$ & 0.044 \\
RPE Pre Test & $6.29(0.47)$ & $6.33(1.29)$ & 0.897 \\
RPE Post Test & $7.71(1.27)$ & $9.77(2.40)$ & 0.008 \\
\hline
\end{tabular}




\subsection{Gait}

\subsubsection{Phase Coordination Index}

There was a main effect of age group for PCI with OA demonstrating a significantly larger PCI as compared to YA $(\mathrm{F}(1,25)=4.46, \mathrm{p}=0.013)$. There was also a main effect between the three walking conditions for PCI $(\mathrm{F}(2,24)=57.55, \mathrm{p}<0.001)$. Post-hoc comparisons using Tukey HSD test indicated the mean value for the normal walking condition $(2.44 \pm 0.85)$ was significantly lower than DT $(3.20 \pm 0.75)$ and fast walk $(3.25 \pm 0.78)$ conditions; however, DT and fast walk were not different. Furthermore, there was no age group by walking condition interaction $(\mathrm{F}(2,24)=0.788, \mathrm{p}=0.466)$, indicating OA demonstrated a greater PCI (e.g. gait was less coordinated) across all conditions as compared to YA.

\subsubsection{Additional gait metrics}

Additional gait metrics showed varying levels of significance between groups, suggesting that OA had an overall decrease in gait performance. Specifically, we report a main effect of age group (Table 2) for all conditions except foot strike angle $\mathrm{CoV}$, and a significant main effect of walking condition (Table 3) for all gait metrics variables. There was also a significant condition by age group interaction for the following variables: mean gait speed $(F(2,26)=7.739, \mathrm{p}=$ $0.002)$, mean stride length $(\mathrm{m})(\mathrm{F}(2,26)=4.10, \mathrm{p}=0.028)$, mean stance $(\% \mathrm{GCT})(\mathrm{F}(2,26)=$ $5.354, \mathrm{p}=0.011)$, stance $(\% \mathrm{GCT}) \operatorname{CoV}(\mathrm{F}(2,26)=3.883, \mathrm{p}=0.033)$, mean swing $(\% \mathrm{GCT})$ $(\mathrm{F}(2,26)=5.356, \mathrm{p}=0.011)$, swing $(\% \mathrm{GCT}) \operatorname{CoV}(\mathrm{F}(2,26)=3.981, \mathrm{p}=0.031)$, mean single limb support $(\% \mathrm{GCT})(\mathrm{F}(2,26)=5.15, \mathrm{p}=0.013)$, single limb support $(\% \mathrm{GCT}) \operatorname{CoV}(\mathrm{F}(2,26)=3.66$, $\mathrm{p}=0.040)$, mean foot strike angle (degrees) $(\mathrm{F}(2,26)=5.24, \mathrm{p}=0.012)$, and mean double support $(\% \mathrm{GCT})(\mathrm{F}(2,26)=3.688, \mathrm{p}=0.039)$. Of the metrics that demonstrated a group by 
condition effect, the significant interactions were either between the normal and DT walk or normal and fast walk.

Table 2. Gait variables separated by group. Values are mean (SD); $p$ values refer to the main effect across age groups. Meters Per Second $=\mathrm{m} / \mathrm{s}$, Percent Gait Cycle Time $=\% \mathrm{GCT}$, coefficient of variation $=\mathrm{CoV}$.

\begin{tabular}{lllll}
\hline & & Young Adult & Old Adult & \\
Walking Variables & & Mean \pm SD & Mean \pm SD & $p$ value \\
\hline Phase Coordination Index & $\%$ & $2.63 \pm 0.66$ & $3.30 \pm 0.94$ & $<0.001$ \\
Gait Speed (m/s) & Mean & $1.42 \pm 0.32$ & $1.20 \pm 0.33$ & 0.002 \\
& CoV & $0.04 \pm 0.01$ & $0.05 \pm 0.02$ & 0.001 \\
Stance (\%GCT) & Mean & $58.51 \pm 1.88$ & $60.31 \pm 2.67$ & $<0.001$ \\
& CoV & $0.01 \pm 0.00$ & $0.01 \pm 0.00$ & $<0.001$ \\
Swing (\%GCT) & Mean & $41.49 \pm 1.88$ & $39.69 \pm 2.67$ & $<0.001$ \\
& CoV & $0.02 \pm 0.00$ & $0.02 \pm 0.01$ & $<0.001$ \\
Cadence Left (steps/min) & Mean & $124.91 \pm 15.64$ & $116.75 \pm 16.08$ & 0.019 \\
& CoV & $0.02 \pm 0.01$ & $0.03 \pm 0.01$ & 0.003 \\
Cadence Right (steps/min) & Mean & $124.87 \pm 15.57$ & $116.73 \pm 16.05$ & 0.019 \\
& CoV & $0.02 \pm 0.01$ & $0.03 \pm 0.01$ & $<0.001$ \\
Double Support (\%GCT) & Mean & $17.21 \pm 3.35$ & $20.70 \pm 5.19$ & $<0.001$ \\
& CoV & $0.05 \pm 0.02$ & $0.06 \pm 0.02$ & 0.059 \\
Foot Strike Angle (Degrees) & Mean & $21.40 \pm 3.81$ & $15.86 \pm 4.46$ & $<0.001$ \\
& CoV & $0.07 \pm 0.02$ & $0.14 \pm 0.21$ & 0.035 \\
Toe Off Angle (Degrees) & Mean & $37.59 \pm 3.58$ & $33.21 \pm 5.81$ & $<0.001$ \\
& CoV & $0.04 \pm 0.01$ & $0.05 \pm 0.02$ & 0.042 \\
Single Limb Support (\%GCT) & Mean & $41.48 \pm 1.86$ & $39.68 \pm 2.67$ & $<0.001$ \\
& CoV & $0.02 \pm 0.00$ & $0.02 \pm 0.01$ & $<0.001$ \\
Stride Length (m) & Mean & $1.35 \pm 0.18$ & $1.21 \pm 0.21$ & 0.015 \\
& CoV & $0.03 \pm 0.01$ & $0.03 \pm 0.01$ & 0.004 \\
Gait Cycle Duration (s) & Mean & $0.98 \pm 0.11$ & $1.05 \pm 0.14$ & 0.025 \\
Step Duration (s) & CoV & $0.02 \pm 0.01$ & $0.03 \pm 0.01$ & 0.008 \\
& Mean & $0.49 \pm 0.06$ & $0.52 \pm 0.07$ & 0.028 \\
& CoV & $0.02 \pm 0.01$ & $0.03 \pm 0.02$ & 0.009 \\
\hline & & & & \\
& & & & \\
& & & & \\
& & & & \\
& & &
\end{tabular}


Table 3. Gait variables separated by condition. Values are mean (SD); $p$ values refer to the main effect across condition. Superscript letters indicate connected letter display; levels not connected by the same letter indicate significant differences. Meters Per Second $=\mathrm{m} / \mathrm{s}$, Percent Gait Cycle Time $=\% \mathrm{GCT}$, coefficient of variation $=\mathrm{CoV}$.

\begin{tabular}{|c|c|c|c|c|c|}
\hline Walking Variables & & $\begin{array}{l}\text { Normal } \\
\text { Mean } \pm \text { SD }\end{array}$ & $\begin{array}{l}\text { Fast } \\
\text { Mean } \pm \text { SD }\end{array}$ & $\begin{array}{l}\text { Dual Task } \\
\text { Mean } \pm \text { SD }\end{array}$ & $\begin{array}{l}p \\
\text { values }\end{array}$ \\
\hline $\begin{array}{l}\text { Phase Coordination } \\
\text { Index }\end{array}$ & $\%$ & $2.44 \pm 0.85^{\mathrm{B}}$ & $3.25 \pm 0.78^{\mathrm{A}}$ & $3.2 \pm 0.75^{\mathrm{A}}$ & $<0.001$ \\
\hline Gait Speed (m/s) & $\begin{array}{l}\text { Mean } \\
\mathrm{CoV}\end{array}$ & $\begin{array}{l}1.19 \pm 0.21^{\mathrm{B}} \\
0.04 \pm 0.01^{\mathrm{B}}\end{array}$ & $\begin{array}{l}1.65 \pm 0.30^{\mathrm{A}} \\
0.04 \pm 0.01^{\mathrm{B}}\end{array}$ & $\begin{array}{l}1.08 \pm 0.02^{\mathrm{B}} \\
0.05 \pm 0.03^{\mathrm{A}}\end{array}$ & $\begin{array}{l}<0.001 \\
0.012\end{array}$ \\
\hline Stance $(\% \mathrm{GCT})$ & $\begin{array}{l}\text { Mean } \\
\text { CoV }\end{array}$ & $\begin{array}{l}60.08 \pm 1.88^{\mathrm{A}} \\
0.01 \pm 0.00^{\mathrm{A}}\end{array}$ & $\begin{array}{l}57.56 \pm 2.43^{\mathrm{B}} \\
0.01 \pm 0.00^{\mathrm{A}}\end{array}$ & $\begin{array}{l}60.69 \pm 1.93^{\mathrm{A}} \\
0.01 \pm 0.01{ }^{\mathrm{A}}\end{array}$ & $\begin{array}{l}<0.001 \\
<0.001\end{array}$ \\
\hline Swing (\%GCT) & $\begin{array}{l}\text { Mean } \\
\text { CoV }\end{array}$ & $\begin{array}{l}39.92 \pm 1.88^{\mathrm{B}} \\
0.02 \pm 0.011^{\mathrm{A}}\end{array}$ & $\begin{array}{l}42.44 \pm 2.43^{\mathrm{A}} \\
0.02 \pm 0.00^{\mathrm{A}}\end{array}$ & $\begin{array}{l}39.31 \pm 1.93^{\mathrm{B}} \\
0.02 \pm 0.01^{\mathrm{A}}\end{array}$ & $\begin{array}{l}<0.001 \\
0.003\end{array}$ \\
\hline $\begin{array}{l}\text { Cadence Left } \\
\text { (steps/min) }\end{array}$ & Mean & $\begin{array}{l}115.41 \pm \\
10.55^{\mathrm{B}}\end{array}$ & $\begin{array}{l}136.25 \pm \\
14.66^{\mathrm{A}}\end{array}$ & $\begin{array}{l}110.41 \pm \\
10.02^{\mathrm{C}}\end{array}$ & $<0.001$ \\
\hline $\begin{array}{l}\text { Cadence Right } \\
\text { (steps/min) }\end{array}$ & $\begin{array}{l}\text { CoV } \\
\text { Mean }\end{array}$ & $\begin{array}{l}0.02 \pm 0.01^{\mathrm{B}} \\
115.38 \pm \\
10.54^{\mathrm{B}}\end{array}$ & $\begin{array}{l}0.02 \pm 0.01^{\mathrm{B}} \\
136.14 \pm \\
14.63^{\mathrm{A}}\end{array}$ & $\begin{array}{l}0.03 \pm 0.02^{\mathrm{A}} \\
110.45 \pm \\
10.04^{\mathrm{C}}\end{array}$ & $\begin{array}{l}0.001 \\
<0.001\end{array}$ \\
\hline $\begin{array}{l}\text { Double Support } \\
(\% \mathrm{GCT})\end{array}$ & $\begin{array}{l}\text { CoV } \\
\text { Mean }\end{array}$ & $\begin{array}{l}0.02 \pm 0.01^{\mathrm{B}} \\
20.18 \pm 3.74^{\mathrm{A}}\end{array}$ & $\begin{array}{l}0.02 \pm 0.01^{\mathrm{A}} \\
15.51 \pm 4.41^{\mathrm{B}}\end{array}$ & $\begin{array}{l}0.03 \pm 0.02^{\mathrm{C}} \\
21.36 \pm 3.86^{\mathrm{A}}\end{array}$ & $\begin{array}{l}0.005 \\
<0.001\end{array}$ \\
\hline $\begin{array}{l}\text { Foot Strike Angle } \\
\text { (Degrees) }\end{array}$ & $\begin{array}{l}\text { CoV } \\
\text { Mean }\end{array}$ & $\begin{array}{l}0.05 \pm 0.02^{\mathrm{B}} \\
17.80 \pm 4.48^{\mathrm{B}}\end{array}$ & $\begin{array}{l}0.07 \pm 0.02^{\mathrm{A}} \\
21.02 \pm 5.07^{\mathrm{A}}\end{array}$ & $\begin{array}{l}0.06 \pm 0.02^{\text {В }} \\
16.79 \pm 4.52^{\text {В }}\end{array}$ & $\begin{array}{l}0.007 \\
<0.001\end{array}$ \\
\hline $\begin{array}{l}\text { Toe Off Angle } \\
\text { (Degrees) }\end{array}$ & $\begin{array}{l}\text { CoV } \\
\text { Mean }\end{array}$ & $\begin{array}{l}0.086 \pm 0.01^{\mathrm{A}} \\
34.93 \pm 4.91 \\
\mathrm{AB}\end{array}$ & $\begin{array}{l}0.089 \pm 0.01^{\mathrm{A}} \\
37.56 \pm 5.10^{\mathrm{A}}\end{array}$ & $\begin{array}{l}0.09 \pm 0.01^{\mathrm{A}} \\
33.49 \pm 4.52^{\mathrm{B}}\end{array}$ & $\begin{array}{l}0.577 \\
<0.001\end{array}$ \\
\hline $\begin{array}{l}\text { Single Limb Support } \\
(\% \text { GCT })\end{array}$ & $\begin{array}{l}\text { CoV } \\
\text { Mean }\end{array}$ & $\begin{array}{l}0.05 \pm 0.02^{\mathrm{A}} \\
39.33 \pm 1.93^{\mathrm{B}}\end{array}$ & $\begin{array}{l}0.03 \pm 0.01^{\mathrm{B}} \\
42.41 \pm 2.42^{\mathrm{A}}\end{array}$ & $\begin{array}{l}0.05 \pm 0.02^{\mathrm{A}} \\
39.91 \pm 1.89^{\mathrm{B}}\end{array}$ & $\begin{array}{l}<0.001 \\
<0.001\end{array}$ \\
\hline Stride Length (m) & $\begin{array}{l}\mathrm{CoV} \\
\text { Mean } \\
\mathrm{CoV}\end{array}$ & $\begin{array}{l}0.02 \pm 0.00^{\mathrm{A}} \\
1.23 \pm 0.15^{\mathrm{B}} \\
0.02 \pm 0.01^{\mathrm{B}}\end{array}$ & $\begin{array}{l}0.02 \pm 0.00^{\mathrm{A}} \\
1.45 \pm 0.19^{\mathrm{A}} \\
0.03 \pm 0.01^{\mathrm{AB}}\end{array}$ & $\begin{array}{l}0.02 \pm 0.01^{\mathrm{A}} \\
1.16 \pm 0.16^{\mathrm{B}} \\
0.03 \pm 0.01^{\mathrm{A}}\end{array}$ & $\begin{array}{l}0.001 \\
<0.001 \\
<0.001\end{array}$ \\
\hline $\begin{array}{l}\text { Gait Cycle Duration } \\
\text { (s) }\end{array}$ & Mean & $\begin{array}{l}1.05 \pm 0.09^{\mathrm{A}} \\
0.02 \pm 0.01^{\mathrm{B}}\end{array}$ & $0.89 \pm 0.10^{\mathrm{B}}$ & $\begin{array}{l}1.10 \pm 0.10^{\mathrm{A}} \\
0.03 \pm 0.02^{\mathrm{A}}\end{array}$ & $<0.001$ \\
\hline Step Duration (s) & $\begin{array}{l}\text { Mean } \\
\mathrm{CoV}\end{array}$ & $\begin{array}{l}0.52 \pm 0.05^{\mathrm{A}} \\
0.02 \pm 0.01^{\mathrm{B}}\end{array}$ & $\begin{array}{l}0.45 \pm 0.05^{\mathrm{B}} \\
0.03 \pm 0.01^{\mathrm{AB}}\end{array}$ & $\begin{array}{l}0.55 \pm 0.05^{\mathrm{A}} \\
0.03 \pm 0.02^{\mathrm{A}}\end{array}$ & $\begin{array}{l}<0.001 \\
0.008\end{array}$ \\
\hline
\end{tabular}




\subsection{Postural Stability}

The postural stability metrics revealed inconsistent results between age groups, suggesting that healthy OA have reduced postural stability in some sway domains however they are not consistently seen in all domains (Table 4). Specifically, for time-domain measures there was a main effect of age group for RMS $(\mathrm{m} / \mathrm{s} 2)(\mathrm{F}(1,24)=4.830, \mathrm{p}=0.038)$ and mean velocity $(\mathrm{m} / \mathrm{s})(\mathrm{F}(1,26)=4.823, \mathrm{p}=0.038)$. However, the additional time-domain measures did not reach significance: path length $(\mathrm{m} / \mathrm{s} 2)(\mathrm{F}(1,26)=0.286, \mathrm{p}=0.597)$ and range $(\mathrm{m} / \mathrm{s} 2)(\mathrm{F}(1,26)=3.660$, $p=0.067)$. Additionally, frequency-domain and jerk measures did not reach significance: $95 \%$ ellipse sway area $(\mathrm{m} 2 / \mathrm{s} 4)(\mathrm{F}(1,24)=2.402, \mathrm{p}=0.133)$, jerk $(\mathrm{m} 2 / \mathrm{s} 5)(\mathrm{F}(1,25)=1.358, \mathrm{p}=0.255)$. Although many of the sway metrics did not reach significance within their specific domains, it is clear when assessing the results that OA have greater variability within the metrics which likely resulted in comparisons not reaching statistical significance. Moreover, as a group, the OA demonstrated increased values in all measures suggesting that their postural stability is comparably worse than their younger counterparts.

Table 4. Sway variables separated by group. Values are mean (SD); $p$ values refer to the main effect across age groups. $95 \%$ ellipse sway area $=$ The area of an ellipse covering $95 \%$ of the sway angle in the coronal and sagittal planes, Jerk $=$ Smoothness of sway from the time derivative of the sway path in the transverse plane (top view looking down), RMS sway $=$ The root mean square (RMS) of the sway angle in both the coronal and sagittal planes, Range $=$ Total range of the sway path in the transverse plane, Path length = Total length of the sway path in the transverse plane, Mean Velocity = Mean velocity of the sway path in the transverse plane.

\begin{tabular}{lllll}
\hline & & Young Adult & Old Adult \\
Sway Variables & Sway Domain & Mean \pm SD & Mean \pm SD & $p$ value \\
\hline $95 \%$ ellipse sway area $\left(\mathrm{m}^{2} / \mathrm{s}^{4}\right)$ & Frequency & $0.17 \pm 0.15$ & $0.43 \pm 0.98$ & 0.133 \\
Jerk $\left(\mathrm{m}^{2} / \mathrm{s}^{5}\right)$ & Jerk & $7.02 \pm 8.75$ & $16.80 \pm 37.74$ & 0.255 \\
RMS Sway $\left(\mathrm{m} / \mathrm{s}^{2}\right)$ & Time & $0.14 \pm 0.07$ & $0.17 \pm 0.15$ & 0.038 \\
Range $\left(\mathrm{m} / \mathrm{s}^{2}\right)$ & Time & $0.73 \pm 0.35$ & $1.05 \pm 1.06$ & 0.067 \\
Path Length $\left(\mathrm{m} / \mathrm{s}^{2}\right)$ & Time & $15.49 \pm 9.66$ & $20.17 \pm 24.05$ & 0.597 \\
Mean Velocity $(\mathrm{m} / \mathrm{s})$ & Time & $0.21 \pm 0.10$ & $0.26 \pm 0.15$ & 0.038 \\
\hline
\end{tabular}




\subsection{Turning}

\subsubsection{Dynamic Turning}

There was a main effect of age group for turn duration with OA demonstrating a significantly longer turn duration compared to YA $(F(1,27)=16.14, \mathrm{p}<0.001)$ (Table 5). Additionally, there was a main effect of walking condition on turn duration $(F(1,26)=43.70, p<$ 0.001) (Table 6). Post-hoc analysis corrected for multiple comparison using Tukey-HSD test indicated mean values for the fast walking condition $(1.80 \pm 0.33)$ were significantly faster than the normal walk $(2.16 \pm 0.33)$ and DT walk $(2.19 \pm 0.43)$; however, normal and DT walk were not different. Furthermore, there were no age group by dynamic turn walking condition interaction $(\mathrm{F}(2,26)=1.45, \mathrm{p}=0.254)$, indicating OAs demonstrated overall slower turning across all conditions as compared to YA.

Additional dynamic turning metrics showed consistent levels of significance between groups, suggesting that OA had overall decreased turning performance. Specifically, we report a main effect of age group for the following metrics: turn velocity (degree/s) $(\mathrm{F}(1,27)=15.59, \mathrm{p}=$ $0.0005)$ and steps in turn $(\#)(F(1,27)=4.94, p=0.035)$. Furthermore, we report a main effect of condition for turn velocity (degrees/s) $(\mathrm{F}(2,26)=37.64, \mathrm{p}=0.0001)$, and turn angle (degree) $(\mathrm{F}(2,26)=7.87, \mathrm{p}=0.0021)$. Main effect of condition results indicate that participants turned faster during the fast walking condition and that turn angle was influenced by walking condition; specifically, during the fast walking condition participants showed a significantly increased turn angle compared to the DT condition.

\subsubsection{Stable Turning}

The results for stable turning revealed a significant main effect of age group for all stable turning metrics (Table 5). We report group differences for: turn duration $(F(1,27)=11.69, \mathrm{p}=$ 
$0.002)$, turn angle (degrees) $(\mathrm{F}(1,27)=14.32, \mathrm{p}<0.001)$, turn velocity $($ degrees $/ \mathrm{s})(\mathrm{F}(1,27)=$ 20.41, $\mathrm{p}<0.001)$ and turn angle accuracy $(\mathrm{F}(1,27)=14.32, \mathrm{p}<0.001)$. The stable turning results indicated that $\mathrm{OA}$ take significantly longer to turn and with a reduced velocity compared to their younger counterparts. Additionally, when assessing the achieved turning accuracy of $360^{\circ}$, YA over turn the desired amount by an average of $22.89^{\circ} \pm 10.25^{\circ}$ while OA on average over turn less with an average of $8.55^{\circ} \pm 10.15^{\circ}$. Specifically, we report a change in stable turning characteristics with advancing age, such that their turning resembled a more cautious approach to the required action.

Table 5. Dynamic and stable turn variables separated by group. Values are mean (SD); $p$ values refer to the main effect across age groups.

\begin{tabular}{llll}
\hline Turn Variables & Young Adult & Old Adult & \\
Dynamic $\left(180^{\circ}\right.$ Turns) & Mean \pm SD & Mean \pm SD & $p$ value \\
\hline Turn Duration (s) & $1.84 \pm 0.23$ & $2.25 \pm 0.43$ & $<0.001$ \\
Turn Velocity (degree/s) & $232.02 \pm 45.20$ & $184.87 \pm 46.11$ & $<0.001$ \\
Steps in Turn (\#) & $3.60 \pm 0.41$ & $3.94 \pm 0.60$ & 0.003 \\
\hline Stable $\left(360^{\circ}\right.$ Turns) & & & \\
\hline Turn Angle (degree) & $382.54 \pm 10.10$ & $368.53 \pm 10.46$ & $<0.001$ \\
Turn Duration (s) & $1.97 \pm 0.29$ & $3.06 \pm 1.10$ & $<0.001$ \\
Turn Velocity (degree/s) & $360.48 \pm 70.18$ & $233.51 \pm 77.71$ & $<0.001$ \\
Turn angle accuracy & $22.89 \pm 10.25$ & $8.55 \pm 10.14$ & $<0.001$ \\
\hline
\end{tabular}

Table 6. Dynamic turn variables separated by condition. Values are mean (SD); $p$ values refer to the main effect across condition. Superscript letters indicate connected letter display; levels not connected by the same letter indicate significant differences. Turn Angle $=$ The rotational angle of the turn, Turn Duration $=$ The duration of the turn, Turn Velocity $=$ The peak angular velocity of the turn.

\begin{tabular}{lllll}
\hline & Normal & Fast & Dual Task & \\
Dynamic Turn Variables & Mean \pm SD & Mean \pm SD & Mean \pm SD & $p$ value \\
\hline Turn Angle (degree) & $185.01 \pm 4.24^{\mathrm{A}}$ & $187.62 \pm 6.76^{\mathrm{B}}$ & $183.02 \pm 5.77^{\mathrm{AB}}$ & 0.002 \\
Turn Duration (s) & $2.16 \pm 0.33^{\mathrm{A}}$ & $1.80 \pm 0.33^{\mathrm{B}}$ & $2.19 \pm 0.43^{\mathrm{A}}$ & $<0.001$ \\
Turn Velocity (degree/s) & $191.00 \pm 37.17^{\mathrm{A}}$ & $245.94 \pm 53.94^{\mathrm{B}}$ & $185.96 \pm 38.40^{\mathrm{B}}$ & $<0.001$ \\
\hline
\end{tabular}




\subsection{Cortical Silent Period (cSP)}

Strength measures for the VMO were statistically different between age groups with YA demonstrating an overall greater leg strength $(\mathrm{t}(56)=-5.313, \mathrm{p}<0.001)$. No significant difference was found for RMT of either leg, although a t-test demonstrated convincing trends for OA requiring higher stimulator intensity in eliciting MEPs (left VMO, $\mathrm{t}(27)=1.583, \mathrm{p}=0.125$; right $\mathrm{VMO}, \mathrm{t}(27)=1.99, \mathrm{p}<0.056$ ). Silent period duration analysis confirmed a hemisphere by age group interaction: $(\mathrm{F}(1,24)=14.347, \mathrm{p}<0.001)$ with post-hoc analysis showing OA had a significantly shorter $\mathrm{cSP}$ in the non-dominant, right motor cortex $(\mathrm{t}(27)=2.072, \mathrm{p}=0.048)$. All other main effects were non-significant (F's $\leq 1.78$, p's $\geq 0.19$ ).

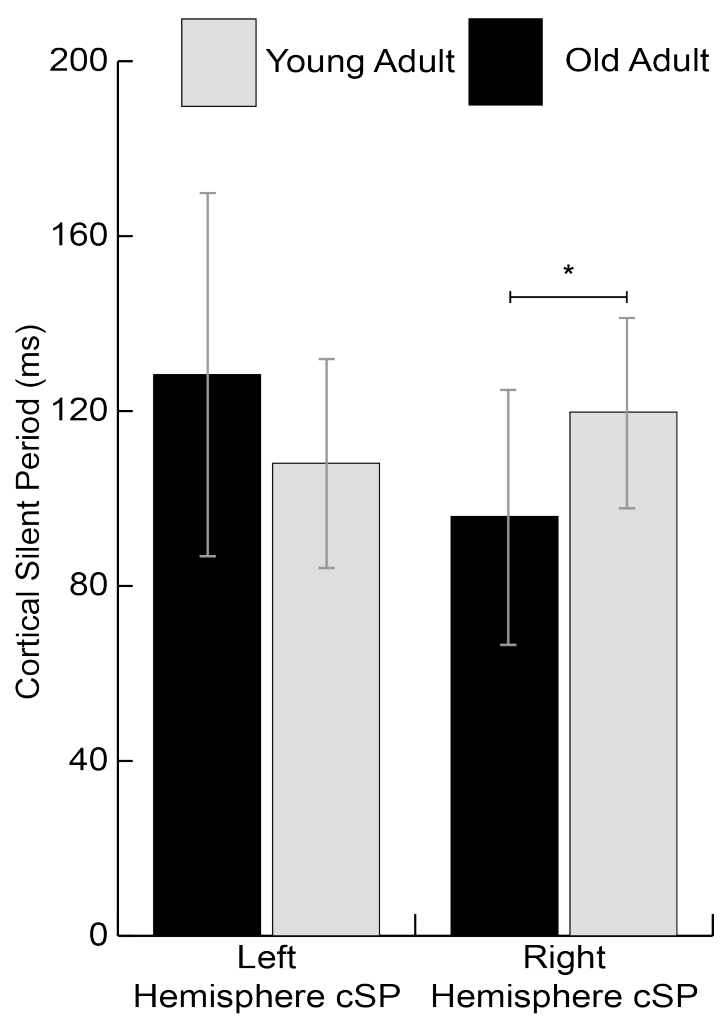

Figure 2. Cortical silent period (cSP) by group and hemisphere indicating a significant difference between the right hemisphere for young and older adults. $*=\mathrm{P}<0.05$. 


\subsection{Correlations}

\subsubsection{Coordination and cSP durations}

PCI was significantly correlated with cSP duration for both hemispheres in the YA's (right hemisphere, $r=0.51, p=0.06$; left hemisphere, $r=0.70, p=0.01$ : Figure 3). Conversely, OA demonstrated a significant, negative relationship between PCI and right hemisphere cSP $(r=$ $-0.45 ; \mathrm{p}=0.12)$, but a non-significant relationship between PCI and left hemisphere cSP $(r=-$ $0.45 ; \mathrm{p}=0.11)$. As shown in Figure $4 \mathrm{a}$, the strength of these correlations was significantly different between age groups for both hemispheres (right, $F(1,4)=246.74, p<0.001$; left, $F(1,4)$ $=40.29 ; \mathrm{p}=0.003$ ). These results indicate that longer $\mathrm{cSPs}$ (i.e. greater motor cortex inhibition) are associated with greater PCI values (i.e. decreased coordination) in the YA, while longer cSPs in OA are related to reduced PCI values (i.e. increased coordination).

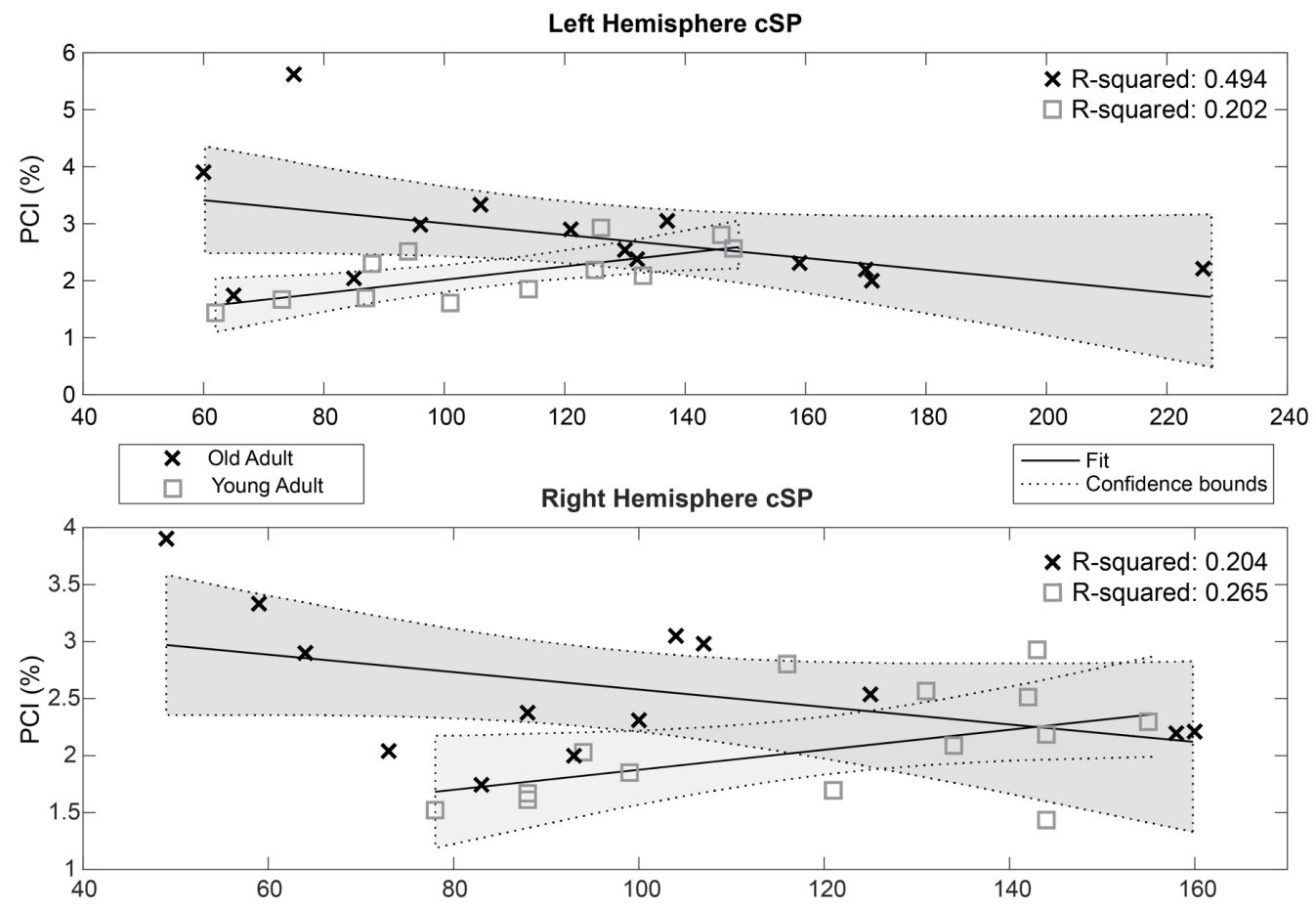

Figure 3. Correlation of normal walk phase coordination index (PCI) and cortical silent period (cSP) duration. Significant, positive correlations were observed between variables for young adults in the right $(\mathrm{r}=0.37, \mathrm{p}=0.016)$ and left hemisphere $(\mathrm{r}=0.54, \mathrm{p} \leq 0.001)$. Older adults demonstrated a significant, negative relationship between PCI and the right $(r=-0.32 ; p=$ $0.042)$, but not the left hemisphere $(r=-0.23 ; p=0.148)$. 


\subsubsection{Gait variability and cSP durations}

Two variability metrics revealed significant correlations of the secondary gait metrics. The YAs demonstrated a significant positive correlation between left hemisphere cSP and stance $(\% \mathrm{GCT}) \mathrm{CoV}(\mathrm{r}=0.48 ; \mathrm{p}=0.003)$ and approached significance for the right hemisphere cSP and stance $(\% \mathrm{GCT}) \mathrm{CoV}(\mathrm{r}=0.29 ; \mathrm{p}=0.062)$. The OA had no significant associations for left hemisphere cSP and stance $(\% \mathrm{GCT}) \mathrm{CoV}(\mathrm{r}=-0.005 ; \mathrm{p}=0.977)$ or the right hemisphere cSP and stance $(\% \mathrm{GCT}) \mathrm{CoV}(\mathrm{r}=-0.20 ; \mathrm{p}=0.196)$. Nonetheless, Figure $4 \mathrm{~b}$ shows the strength of these correlations were significantly different between age groups for both hemispheres (right, $F(1,4)=9.33 ; p=0.038 ;$ left, $F(1,4)=21.40 ; p=0.001)$. Swing $(\% G C T)$ CoV for the YA group was significantly correlated to left hemisphere $\operatorname{cSP}(r=0.56 ; \mathrm{p}<0.001)$ while the right hemisphere cSP approached significance $(r=0.30 ; p=0.055)$. Left hemisphere cSP and swing $(\% \mathrm{GCT}) \mathrm{CoV}$ for the OA was not significantly correlated $(\mathrm{r}=-0.05 ; \mathrm{p}=0.734)$ but trended towards significance in the right hemisphere $(r=-0.28 ; \mathrm{p}=0.068)$. Figure $4 \mathrm{c}$ illustrates how the strength of these correlations were also significantly different between age groups and hemispheres: (right, $\mathrm{F}(1,4)=16.44, \mathrm{p}=0.015$; left, $\mathrm{F}(1,4)=19.23, \mathrm{p}=0.012$ ). For both stance and swing (\%GCT) $\mathrm{CoV}$ measures, these results suggest longer silent periods are associate with increased gait variability in YA while longer silent periods are associated with decreased variability for OA. 


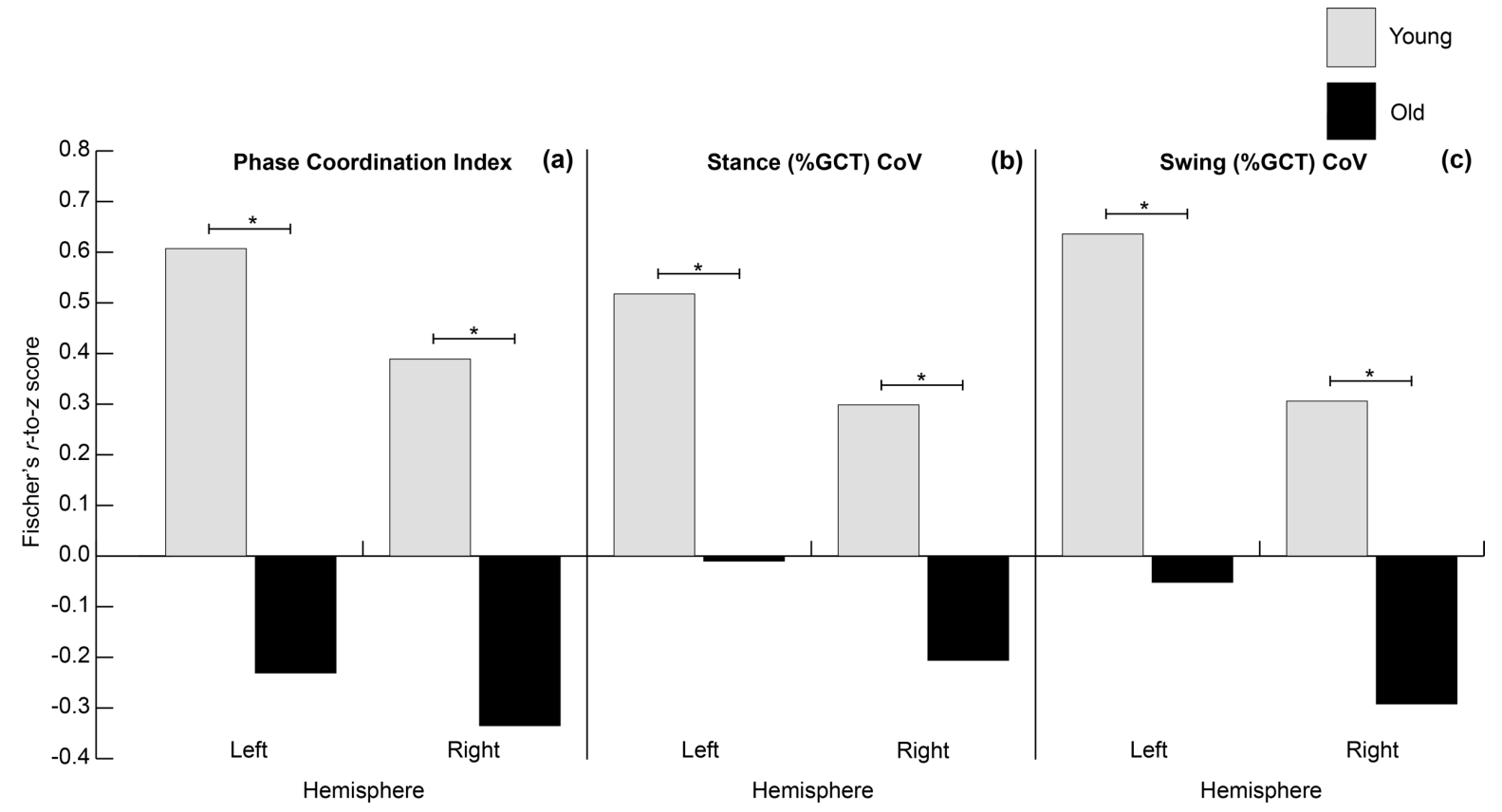

Figure 4. Fisher r-to- $Z$ transforms demonstrating group differences in the strength of associations between (A) phase coordination index (PCI) and cortical silent period (cSP), (B) stance phase variability and cSP, and (C) swing phase variability and cSP. Significant differences between groups were seen in both hemispheres for the gait variables shown, indicating opposing relationships between cSP duration and gait coordination or variability in young and older adults. Percent Gait Cycle Time $=\%$ GCT, coefficient of variation $=\mathrm{CoV}$. 


\section{DISCUSSION}

The primary aim of this study was to assess the effects of aging on cortical inhibition, gait coordination and the hypothesized association between these measures. Gait measurements were derived during three different walking conditions of over ground walking using wireless inertial sensors. Results demonstrated that gait coordination, variability, postural stability, and both dynamic and stable turning are disrupted in OA resulting in an overall decrease in mobility performance compared to YA. Motor cortex inhibition was measured via the cSP of knee extensor muscles and was reduced in the right hemisphere of OA. Furthermore, measures of gait coordination and variability demonstrated a positive relationship with cSP in the YA, while there was a negative correlation of $\mathrm{cSP}$ with lower extremity coordination in OA. These results suggest a fundamental difference in the relationship between motor cortex inhibition and lower extremity control with age; YA are better able to maintain lower extremity coordination and variability with reduced cortical inhibition, whereas OA with increased cortical inhibition demonstrate better walking performance.

\subsection{Gait Coordination}

The PCI has been shown to be affected by different walking conditions, age, neurologic health status, and is not strongly associated with more traditional mobility outcomes such as changes in gait speed (Meir Plotnik, Bartsch, Zeev, Giladi, \& Hausdorff, 2013). Taken together, these results indicate that PCI analyze is an independent domain of gait and as such PCI could be a potential marker for mobility impairments, and a likelihood of increased fall risk (James, Leveille, Hausdorff, Barton, et al., 2017; James, Leveille, Hausdorff, Travison, et al., 2017; James et al., 2016). To date, the majority of studies have evaluated PCI in older and 
neurologically diseased populations with a primary emphasis on Parkinson's disease (PD). Within these populations, gait coordination has been quantified during slow, normal, and fast walking, as well as DT walking (James et al., 2016; Peterson et al., 2012; Meir Plotnik et al., 2013; M Plotnik, Giladi, \& Hausdorff, 2009; M. Plotnik et al., 2007). It has been reported that PCI significantly increases with typical aging during over ground self-selected pace walking compared to healthy young adults (M. Plotnik et al., 2007). Condition effects have also been observed in healthy young adults. For example, Plotnik et al. (2013) report a significant increase in PCI during slow walking, compared to self-selected walking, which they attribute to an interference with left and right anti-phase step patterns and the possibility of increased cortical input during a slow walking task, thereby limiting the control of central pattern generators (Meir Plotnik et al., 2013). In the current study, PCI was used to evaluate bilateral coordination of gait in three distinct walking conditions (normal, DT, and fast) within healthy young and older individuals. To our knowledge this is the first study comparing all three walking conditions in OA and YA who are all neurologically healthy. As hypothesized, the current results demonstrate that OA exhibited a greater PCI than the YA across all walking conditions, a finding that is consistent with previous research demonstrating decreased inter-limb coordination with age (M. Plotnik et al., 2007). Additionally, there were significant increases in PCI in the fast and DT conditions when compared to the normal walk. These findings contradict prior findings which showed no statistical differences in PCI between self-selected pace and the other two walking conditions. A potential explanation, and limitation of the current study, is that we compared a normal paced six-minute walk to the two-minute DT and fast walks. Conversely, these results could be due to the current sample size which was larger than the two other published studies, or the methods of collections which used force sensitive insoles (Meir Plotnik et al., 2013; M 
Plotnik et al., 2009). The current results demonstrate gait coordination differences independent of walking condition with an overall decrease of coordination within healthy OA.

\subsection{Additional Gait Metrics}

Additional gait metrics were analyzed to assess the various differences between OA and their younger counterparts. These additional metrics included measures of gait variability and more traditional spatiotemporal metrics recorded in the literature. We found OA displayed greater variability in all gait measures except for double support time (\%GCT) $\mathrm{CoV}$ and stride length CoV. Although, the cause of increased variability is relatively unknown, it has been well established that increased gait variability with age is strongly associated with fall risk (Jeffrey M. Hausdorff et al., 2001; Kang \& Dingwell).

Our results are consistent with prior research indicating that variability of step length and time are greater in OA, specifically in an older healthy population and furthermore, the increased variability these two metrics have been observed in clinical studies assessing geriatric and functionally impaired individuals (Callisaya, Blizzard, Schmidt, McGinley, \& Srikanth, 2010). Additionally, increased variability could result in poorer foot placement and the negotiation of objects during ambulation. With the addition of all other variability metrics assessed it is possible that the overall increased variability in OA could demonstrate a decline within central pattern generation and/or declines on central motor control (Callisaya et al., 2010; Jeffrey M. Hausdorff, 2007). Furthermore, it has been speculated that the mechanisms leading to increases in variability could be different between variables although it remains uncertain (Brach, Studenski, Perera, VanSwearingen, \& Newman, 2008). Although, the prior hypothesis remains uncertain, changes to brain structure, sensory input signaling, motor efferent conduction, muscle 
size and fiber type, and disease are probable contributors for the observed increases in variability (Brach et al., 2008; Callisaya et al., 2010).

\subsection{Postural Stability}

Postural stability is comprised of three essential systems working in unison to maintain a steady upright posture. Somatosensory, visual, and vestibular systems all interact together; however, the ability to organize sensory inputs for postural control declines with advancing age (Chaikeeree, Saengsirisuwan, Chinsongkram, \& Boonsinsukh, 2015). The CTSIB functions to dissect the various interactions allowing steady upright posture. Each of the four conditions are specifically designed to measure the interaction of all systems working together and then sequentially tease them apart to understand any discrepancies within specific aspects of balance.

Our results demonstrate consistency with prior studies supporting the concept that balance declines with age. Specifically, we report two metrics with significant group differences in the time-domain. Root mean square (RMS) and mean velocity measures were the only two metrics which reveals significant group differences. It has been proposed that sway area is related to the effectiveness of an individual's postural control system, while the mean velocity is related to the sum of regulatory activity associated with stability (Hufschmidt, Dichgans, Mauritz, \& Hufschmidt, 1980; Martina Mancini et al., 2011). Our results suggested that OA have similar sway area to their younger counterparts however, the OA have significantly increased mean velocity suggesting the amount of regulatory activity to maintain stability is increased resulting in a reduced organization of sensory inputs. We also found larger RMS sway in the OA which is measured as 2D sway variability averaged in both the anterior-posterior and mediallateral directions. The increased RMS sway is thought to reflect noisy somatosensory feedback from joint receptors and muscle proprioception within the postural control loops (Martina 
Mancini et al., 2011). Disruptions to the open postural control loop may result in inaccurate information about body position in relation to its surrounding therefore creating an abnormal internal map of stability limits and overall unsteadiness (Collins, De Luca, Burrows, \& Lipsitz, 1995; Martina Mancini et al., 2011). The current results demonstrate postural stability differences in time-domain measures of balance between OA and their younger counterparts. Suggesting, that OAs are receiving incomplete or inaccurate sensory feedback as measured by RMS and therefore, as a result OA are increasing their mean velocity to maintain an upright posture.

\section{$5.4 \quad$ Turning}

Turning, or the ability to alter locomotion by changing direction is not only a common aspect of walking it is also incredibly important for individual's functional independence. Additionally, the neural control of turning is much more expensive to the nervous system than straight ahead walking which in healthy individuals is primarily controlled at the spinal level. It has been discovered that measures of turning are able to discriminate between fallers from nonfallers, which in aging adults a fall while turning increases their likelihood of a hip fracture compared to a fall while straight ahead walking (Cumming \& Klineberg, 1994; Dite \& Temple, 2002; El-Gohary et al., 2013; Feldman \& Robinovitch, 2007). To date, a majority of the studies assessing turning have been focused on the comparison of elderly fallers and non-fallers, or the assessment of people who have a diagnosed movement disorder or some other form of

neurological disability affecting mobility (El-Gohary et al., 2013; Fino et al., 2018; King et al., 2012; Martina Mancini et al., 2011; Martina Mancini et al., 2016; Spain et al., 2012). However, the current literature lacks objective evidence for age related changes to many turning metrics currently reported in the movement disorders literature. For age related turning changes many 
themes arise, such as differences in turn duration, turn velocity, turn angle, and number of steps within a turn (El-Gohary et al., 2013). Traditionally, age related turning differences have been studied using full 3D motion analysis, and the use of foot constraints to assess the segmental transverse dynamics of the spinal cord while turning (Baird \& Van Emmerik, 2009; Thigpen, Light, Creel, \& Flynn, 2000).

To our knowledge this is the first study to assess healthy age-related differences in turning using wireless inertial sensors to assess many of the same outcome variables which have been tested in recent movement disorders literature. This study also recorded two independent types of turning. Dynamic turning which is a turn associated with a walking bout (typically $180^{\circ}$ ) and stable turning which is associated with a turn that might occur in closed space (typically $360^{\circ}$ ). Regarding dynamic turns, OA in the current study demonstrate similarities in turn duration for elderly fallers and non-fallers (Martina Mancini et al., 2016). The differences seen in the dynamic turning metrics between the two groups suggests a more cautious turning pattern. This observation is made clearer based on the fact that OA have a significantly reduced turning velocity, with an increased turn duration and steps within the turn. Similar results for stable turning were observed. Aside from turn duration which was significantly longer in the OA, all other metrics (turn angle, velocity, and turn accuracy) were decreased in the OA. The assessment of turn angle accuracy results demonstrated that OA were more accurate when asked to complete a $360^{\circ}$ turning task as fast as possible compared to their younger counterparts. It has been reported in the upper extremities that OA demonstrate a significant speed to accuracy trade off, where when asked to complete a task as fast and accurately as possible OA will reduce their speed in order to be more accurate (Brogmus, 1991; G. A. Smith \& Brewer, 1995). Additionally, this could be a result of subconscious remediation of instability for OA. In contrast, YA feel 
comfortable continually maintaining their center of mass over their base of support resulting in less accuracy, merely due to the fact that they are inherently more stable.

The differences in turning between the two groups has been considered a developmental change. This theory is in-line with a hypothesis by VanSant and colleagues suggesting that motor development is a lifelong process, that age-related changes in movement patterns which are not directly related to motor learning may occur throughout the lifespan (VanSant, 1988).

Additionally, the inability to correctly organize various signals both afferent and efferent, can produce a temporal delay, likely subconsciously mediated. However, resulting in a compensatory mechanism of reduced spatiotemporal actions. Moreover, further studies should be conducted to assess the neural control of both dynamic and stable turning to further understand the various mechanisms behind successful and detrimental turns.

\subsection{Cortical silent period/cortical inhibition and age}

Previous studies have reported discrepancies between age and cortical inhibition, demonstrating inhibitory decreases, increases, or no change. Inhibitory differences have been identified in studies assessing the impact of age on motor cortex excitability on various upper extremity muscle groups (Heise et al., 2013; McGinley, Hoffman, Russ, Thomas, \& Clark, 2010;

Oliviero et al., 2006; A. E. Smith, Ridding, Higgins, Wittert, \& Pitcher, 2011). Within this literature, it has been reported that age causes various changes to the inhibitory properties of the motor cortex particularly within the hand and arm regions of the motor cortex. For example, reduced inhibition has been observed when measuring the cSP (Oliviero et al., 2006) and the ipsilateral silent period (Fling \& Seidler, 2012) of the first dorsal interosseous muscle in OA. Conversely, increased short- and long- interval motor cortex inhibition (elicited via paired-pulse TMS) has been shown in the flexor carpi radialis muscle of the non-dominant arm in OA 
(McGinley et al., 2010). In the current study, cSP was used to assess levels of motor cortex inhibition of the VMO in young and older adults. Although there was a lack of group effect for cSP, which did not support our hypothesis that OA would have a significantly shorter cSP than their YA counterparts, there was an age group by hemisphere interaction, indicating a significantly shorter cSP in the non-dominant, right hemisphere of OA.

It is interesting to note that motor cortex inhibition was solely reduced in the nondominant hemisphere of OA. Older adults demonstrate a task-based increase of hand dominance with advancing age, demonstrating an increased reliance on the dominant hemisphere (i.e. left hemisphere in this instance) (Sivagnanasunderam et al., 2015). The current results support a neural mechanism for this behavioral phenomenon indicating that cortical inhibition is maintained within the dominant hemisphere, whereas it is reduced on the non-dominant side. Additionally, these results align with current aging literature postulating that during motor performance tasks OA demonstrate an over activation of the brain. More specifically it has been shown over activation is positively associated with motor performance including static limb coordination accuracy resulting in increased activation corresponding with the motor and sensorimotor regions of the brain (Heuninckx, Wenderoth, \& Swinnen, 2008; Seidler et al., 2010). The documented increases in brain activation are thought to be a compensatory mechanism for the age-related structural and biochemical declines that accompany advancing age (Seidler et al., 2010).

\subsection{Cortical silent period and bilateral coordination}

Recent literature has consistently demonstrated that OA have a reduced capacity and ability to coordinate their movements (Goble et al., 2010; Greene \& Williams, 1996; Heise et al., 2013; Heuninckx, Debaere, Wenderoth, Verschueren, \& Swinnen, 2004; Serrien, Swinnen, \& 
Stelmach, 2000). Older adults also demonstrate decreases in their ability to limit variability during coordinated movements relative to their younger counterparts. In the upper extremities these reductions in coordination typically do not completely limit the completion or performance of the task but instead reduce completion speed and/or accuracy (Heuninckx et al., 2004; Temprado, Vercruysse, Salesse, \& Berton, 2010; Wishart, Lee, Murdoch, \& Hodges, 2000). When assessing the cortical contributions to coordination, tasks requiring bimanual movements in time and space at different speeds or amplitudes cause decreased accuracy and consistency (Levin, Fujiyama, Boisgontier, Swinnen, \& Summers, 2014; Levin, Suy, Huybrechts, Vangheluwe, \& Swinnen, 2004; Serrien \& Swinnen, 1997). Age-related declines of motor cortex inhibition have been associated with declines in motor performance, thus, providing evidence that age related changes to intercortical processing affects motor control (Hortobágyi et al., 2006; Oliviero et al., 2006; Papegaaij et al., 2014). To date most studies assessing inhibition and motor performance have done so through various TMS techniques with a focus on upper extremity manual dexterity, sedentary limb coordination, and static postural stability (Fujiyama et al., 2009; Heise et al., 2013; Levin et al., 2014; Papegaaij et al., 2014).

The study of interlimb coordination has been primarily focused on the upper extremities and there have been few studies assessing how cortical excitation or inhibition affects the lower extremities in combination with the upper extremities (Sohn, Kang, \& Hallett, 2005). It has been established in the upper and lower extremities that cSP is associated with proper interlimb coordination, specifically Fujiyama and colleagues suggest degradation of motor performance (i.e. limb coordination) is associated with a decreased ability to modulate or activate GABAergic circuits (Fujiyama et al., 2009; Levin et al., 2014). In the current study cSP was used to understand the associations between gait coordination and hemispheric inhibition. To our 
knowledge this is the first study to assess the relationships between gait coordination and cortical inhibition and the subsequent effects of age. The current results support previous findings for cSP duration and interlimb coordination in both YA and OA. We report that YA have significantly different cortical regulation of gait coordination (assessed by PCI) than OA. Specifically, we report that a shorter cSP for YA equates to better interlimb coordination. Furthermore, our results are consistent with research indicating that OA demonstrate an inhibitory shift associating longer cSPs to better interlimb coordination (Fujiyama et al., 2009). These results further support the theory that OA have a change in the balance of intrahemispheric inhibition with age. Specifically, OA who are able to maintain inhibition preserve their ability to maintain coordinated control of their legs while ambulating. 


\section{CONCLUSION}

With advancing age mobility is affected in a variety of ways including various walking, postural sway and turning metrics. For gait and dynamic turns, increasing the difficulty of walking tasks has an overall negative effect on gait coordination, variability and traditional metrics, as well as turn velocity and duration. Additionally, when increasing the task difficulty for postural stability, there is a negative effect on jerk, frequency, and time domain metrics. Furthermore, there are numerous differences between age groups demonstrating significantly reduced mobility across a variety of measurements and tests. Specifically, the current results demonstrate reduced bilateral gait coordination and increased gait variability compared to YA. Moreover, our results report reductions in time-domain postural sway metrics including RMS and mean velocity, and finally differences in turning metrics including turn duration, velocity, number of steps, and turn angle accuracy.

In addition, we report a reduction in motor cortex intracortical inhibition of the nondominant hemisphere of older adults. Finally, OA demonstrated an inhibitory shift with age relating to coordination and variability, where increased inhibition was associated with increased gait coordination. This is significantly different from YA who demonstrated worse coordination with increased inhibition. These results compliment and add to the existing literature by demonstrating increased inhibition is related to improved bilateral performance in OA and that increasing task difficulty results in poorer interlimb coordination. Because the current literature lacks substantial evidence assessing cortical control of interlimb coordination of the lower extremities, and because fall risk increases with advancing age, it is important for future research to continue investigating the neural mechanisms underlying successful ambulation specifically in 
the mobility parameters (postural sway and turning) which were not correlated with intracortical inhibition in the current study. 


\section{REFERENCES}

Baird, J. L., \& Van Emmerik, R. E. (2009). Young and older adults use different strategies to perform a standing turning task. Clinical Biomechanics, 24(10), 826-832.

Bhandari, A., Radhu, N., Farzan, F., Mulsant, B. H., Rajji, T. K., Daskalakis, Z. J., \& Blumberger, D. M. (2016). A meta-analysis of the effects of aging on motor cortex neurophysiology assessed by transcranial magnetic stimulation. Clinical Neurophysiology, 127(8), 2834-2845. doi:https://doi.org/10.1016/j.clinph.2016.05.363

Boisgontier, M. P., \& Swinnen, S. P. (2015). Age-related deficit in a bimanual joint position matching task is amplitude dependent. Frontiers in aging neuroscience, 7(162). doi:10.3389/fnagi.2015.00162

Brach, J. S., Berthold, R., Craik, R., VanSwearingen, J. M., \& Newman, A. B. (2001). Gait Variability in Community-Dwelling Older Adults. Journal of the American Geriatrics Society, 49(12), 1646-1650.

Brach, J. S., Studenski, S., Perera, S., VanSwearingen, J. M., \& Newman, A. B. (2008). Stance time and step width variability have unique contributing impairments in older persons. Gait \& Posture, 27(3), 431-439.

Brach, J. S., Studenski, S. A., Perera, S., VanSwearingen, J. M., \& Newman, A. B. (2007). Gait variability and the risk of incident mobility disability in community-dwelling older adults. The Journals of Gerontology Series A: Biological Sciences and Medical Sciences, 62(9), 983-988. 
Brogmus, G. E. (1991). Effects of age and sex on speed and accuracy of hand movements: And the refinements they suggest for Fitts' Law. Paper presented at the Proceedings of the Human Factors Society Annual Meeting.

Callisaya, M. L., Blizzard, L., Schmidt, M. D., McGinley, J. L., \& Srikanth, V. K. (2010). Ageing and gait variability — a population-based study of older people. Age and ageing, 39(2), 191-197.

Cash, R. F., Noda, Y., Zomorrodi, R., Radhu, N., Farzan, F., Rajji, T. K., . . Blumberger, D. M. (2017). Characterization of Glutamatergic and GABA A-Mediated Neurotransmission in Motor and Dorsolateral Prefrontal Cortex Using Paired-Pulse TMS-EEG.

Neuropsychopharmacology, 42(2), 502.

Chaikeeree, N., Saengsirisuwan, V., Chinsongkram, B., \& Boonsinsukh, R. (2015). Interaction of age and foam types used in Clinical Test for Sensory Interaction and Balance (CTSIB). Gait \& Posture, 41(1), 313-315.

Collins, J. J., De Luca, C. J., Burrows, A., \& Lipsitz, L. A. (1995). Age-related changes in openloop and closed-loop postural control mechanisms. Experimental Brain Research, 104(3), 480-492. doi:10.1007/bf00231982

Cumming, R. G., \& Klineberg, R. J. (1994). Fall frequency and characteristics and the risk of hip fractures. Journal of the American Geriatrics Society, 42(7), 774-778.

Di Lazzaro, V., Oliviero, A., Meglio, M., Cioni, B., Tamburrini, G., Tonali, P., \& Rothwell, J. (2000). Direct demonstration of the effect of lorazepam on the excitability of the human motor cortex. Clinical Neurophysiology, 111(5), 794-799.

Dingwell, J. B., Salinas, M. M., \& Cusumano, J. P. (2017). Increased gait variability may not imply impaired stride-to-stride control of walking in healthy older adults: winner: 2013 
Gait and Clinical Movement Analysis Society Best Paper Award. Gait \& Posture, 55, $131-137$.

Dite, W., \& Temple, V. A. (2002). Development of a clinical measure of turning for older adults. American journal of physical medicine \& rehabilitation, 81(11), 857-866.

El-Gohary, M., Pearson, S., McNames, J., Mancini, M., Horak, F., Mellone, S., \& Chiari, L. (2013). Continuous monitoring of turning in patients with movement disability. Sensors, 14(1), 356-369.

Feldman, F., \& Robinovitch, S. N. (2007). Reducing hip fracture risk during sideways falls: evidence in young adults of the protective effects of impact to the hands and stepping. Journal of biomechanics, 40(12), 2612-2618.

Fino, P. C., Parrington, L., Walls, M., Sippel, E., Hullar, T. E., Chesnutt, J. C., \& King, L. A. (2018). Abnormal Turning and Its Association with Self-Reported Symptoms in Chronic Mild Traumatic Brain Injury. Journal of Neurotrauma, 0(0), null. doi:10.1089/neu.2017.5231

Fisher, B. E., Wu, A. D., Salem, G. J., Song, J., Lin, C. H., Yip, J., . . Petzinger, G. (2008). The effect of exercise training in improving motor performance and corticomotor excitability in people with early Parkinson's disease. Arch Phys Med Rehabil, 89(7), 1221-1229. doi:10.1016/j.apmr.2008.01.013

Fling, B. W., Kwak, Y., Peltier, S. J., \& Seidler, R. D. (2012). Differential relationships between transcallosal structural and functional connectivity in young and older adults. Neurobiol Aging, 33(10), 2521-2526. doi:10.1016/j.neurobiolaging.2011.11.018 
Fling, B. W., \& Seidler, R. D. (2012). Fundamental differences in callosal structure, neurophysiologic function, and bimanual control in young and older adults. Cereb Cortex, 22(11), 2643-2652. doi:10.1093/cercor/bhr349

Fujiyama, H., Garry, M. I., Levin, O., Swinnen, S. P., \& Summers, J. J. (2009). Age-related differences in inhibitory processes during interlimb coordination. Brain Research, 1262, 38-47. doi:https://doi.org/10.1016/j.brainres.2009.01.023

Fujiyama, H., Hinder, M. R., Schmidt, M. W., Garry, M. I., \& Summers, J. J. (2012). Agerelated differences in corticospinal excitability and inhibition during coordination of upper and lower limbs. Neurobiology of aging, 33(7), 1484. e1481-1484. e1414.

Garvey, M. A., \& Gilbert, D. L. Transcranial magnetic stimulation in children. European Journal of Paediatric Neurology, 8(1), 7-19. doi:10.1016/j.ejpn.2003.11.002

Goble, D. J., Coxon, J. P., Van Impe, A., De Vos, J., Wenderoth, N., \& Swinnen, S. P. (2010). The neural control of bimanual movements in the elderly: Brain regions exhibiting agerelated increases in activity, frequency-induced neural modulation, and task-specific compensatory recruitment. Human brain mapping, 31(8), 1281-1295.

Greene, L. S., \& Williams, H. G. (1996). Aging and Coordination from the Dynamic Pattern Perspective. In A.-M. Ferrandez \& N. Teasdale (Eds.), Advances in Psychology (Vol. 114, pp. 89-131): North-Holland.

Groppa, S., Oliviero, A., Eisen, A., Quartarone, A., Cohen, L. G., Mall, V., . . Siebner, H. R. (2012). A practical guide to diagnostic transcranial magnetic stimulation: Report of an IFCN committee. Clinical Neurophysiology, 123(5), 858-882. doi:https://doi.org/10.1016/j.clinph.2012.01.010 
Hausdorff, J. M. (2007). Gait dynamics, fractals and falls: Finding meaning in the stride-to-stride fluctuations of human walking. Human movement science, 26(4), 555-589. doi:https://doi.org/10.1016/j.humov.2007.05.003

Hausdorff, J. M., Cudkowicz, M. E., Firtion, R., Wei, J. Y., \& Goldberger, A. L. (1998). Gait variability and basal ganglia disorders: Stride-to-stride variations of gait cycle timing in parkinson's disease and Huntington's disease. Movement Disorders, 13(3), 428-437.

Hausdorff, J. M., Rios, D. A., \& Edelberg, H. K. (2001). Gait variability and fall risk in community-living older adults: A 1-year prospective study. Archives of Physical Medicine and Rehabilitation, 82(8), 1050-1056.

doi:https://doi.org/10.1053/apmr.2001.24893

Heise, K.-F., Zimerman, M., Hoppe, J., Gerloff, C., Wegscheider, K., \& Hummel, F. C. (2013). The Aging Motor System as a Model for Plastic Changes of GABA-Mediated Intracortical Inhibition and Their Behavioral Relevance. The Journal of Neuroscience, 33(21), 9039-9049. doi:10.1523/jneurosci.4094-12.2013

Hermans, L., Levin, O., Maes, C., Van Ruitenbeek, P., Heise, K.-F., Edden, R. A. E., .. . Cuypers, K. GABA levels and measures of intracortical and interhemispheric excitability in healthy young and older adults: a MRS-TMS study. Neurobiology of aging. doi:https://doi.org/10.1016/j.neurobiolaging.2018.01.023

Heuninckx, S., Debaere, F., Wenderoth, N., Verschueren, S., \& Swinnen, S. P. (2004). Ipsilateral coordination deficits and central processing requirements associated with coordination as a function of aging. The Journals of Gerontology Series B: Psychological Sciences and Social Sciences, 59(5), P225-P232. 
Heuninckx, S., Wenderoth, N., \& Swinnen, S. P. (2008). Systems neuroplasticity in the aging brain: recruiting additional neural resources for successful motor performance in elderly persons. Journal of neuroscience, 28(1), 91-99.

Horak, F., King, L., \& Mancini, M. (2015). Role of body-worn movement monitor technology for balance and gait rehabilitation. Phys Ther, 95(3), 461-470. doi:10.2522/ptj.20140253

Horak, F. B. (1987). Clinical measurement of postural control in adults. Physical Therapy, 67(12), 1881-1885.

Hortobágyi, T., del Olmo, M. F., \& Rothwell, J. C. (2006). Age reduces cortical reciprocal inhibition in humans. Experimental Brain Research, 171(3), 322-329. doi:10.1007/s00221-005-0274-9

Hufschmidt, A., Dichgans, J., Mauritz, K.-H., \& Hufschmidt, M. (1980). Some methods and parameters of body sway quantification and their neurological applications. Archiv für Psychiatrie und Nervenkrankheiten, 228(2), 135-150.

James, E. G., Leveille, S. G., Hausdorff, J. M., Barton, B., Cote, S., Karabulut, M., . . Bean, J. F. (2017). Coordination Impairments Are Associated With Falling Among Older Adults. Exp Aging Res, 43(5), 430-439. doi:10.1080/0361073X.2017.1369634

James, E. G., Leveille, S. G., Hausdorff, J. M., Travison, T., Cote, S., Conatser, P., . . Bean, J. F. (2017). Rhythmic Interlimb Coordination Impairments Are Associated With Mobility Limitations Among Older Adults. Exp Aging Res, 43(4), 337-345. doi:10.1080/0361073X.2017.1333819

James, E. G., Leveille, S. G., You, T. J., Hausdorff, J. M., Travison, T., Manor, B., . . Bean, J. F. (2016). Gait coordination impairment is associated with mobility in older adults. Experimental gerontology, 80, 12-16. doi:10.1016/j.exger.2016.04.009 
Kang, H. G., \& Dingwell, J. B. (2007). Separating the effects of age and walking speed on gait variability. Gait \& Posture, 27(4), 572-577. doi:10.1016/j.gaitpost.2007.07.009

King, L. A., Mancini, M., Priest, K., Salarian, A., Rodrigues-de-Paula, F., \& Horak, F. (2012). Do clinical scales of balance reflect turning abnormalities in people with Parkinson's disease? Journal of Neurologic Physical Therapy, 36(1), 25.

Kribus-Shmiel, L., Zeilig, G., Sokolovski, B., \& Plotnik, M. (2018). How many strides are required for a reliable estimation of temporal gait parameters? Implementation of a new algorithm on the phase coordination index. PLoS ONE, 13(2), e0192049. doi:10.1371/journal.pone.0192049

Kujirai, T., Caramia, M., Rothwell, J. C., Day, B., Thompson, P., Ferbert, A., . . Marsden, C. D. (1993). Corticocortical inhibition in human motor cortex. The Journal of physiology, 471(1), 501-519.

Lazzaro, V. D., Restuccia, D., Oliviero, A., Profice, P., Ferrara, L., Insola, A., . . Rothwell, J. C. (1998). Magnetic transcranial stimulation at intensities below active motor threshold activates intracortical inhibitory circuits. Experimental Brain Research, 119(2), 265-268. doi: $10.1007 / \mathrm{s} 002210050341$

Levin, O., Fujiyama, H., Boisgontier, M. P., Swinnen, S. P., \& Summers, J. J. (2014). Aging and motor inhibition: a converging perspective provided by brain stimulation and imaging approaches. Neuroscience \& Biobehavioral Reviews, 43, 100-117.

Levin, O., Suy, E., Huybrechts, J., Vangheluwe, S., \& Swinnen, S. P. (2004). Bimanual coordination involving homologous and heterologous joint combinations: when lower stability is associated with higher flexibility. Behavioural brain research, 152(2), 437445. 
Lord, S., Howe, T., Greenland, J., Simpson, L., \& Rochester, L. (2011). Gait variability in older adults: a structured review of testing protocol and clinimetric properties. Gait Posture, 34(4), 443-450. doi:10.1016/j.gaitpost.2011.07.010

Maki, B. E. (1997). Gait changes in older adults: predictors of falls or indicators of fear? Journal of the American Geriatrics Society, 45(3), 313-320.

Mancini, M., Horak, F. B., Zampieri, C., Carlson-Kuhta, P., Nutt, J. G., \& Chiari, L. (2011). Trunk accelerometry reveals postural instability in untreated Parkinson's disease. Parkinsonism \& related disorders, 17(7), 557-562.

Mancini, M., King, L., Salarian, A., Holmstrom, L., McNames, J., \& Horak, F. B. (2011). Mobility Lab to Assess Balance and Gait with Synchronized Body-worn Sensors. J Bioeng Biomed Sci, Suppl 1, 007. doi:10.4172/2155-9538.S1-007

Mancini, M., Salarian, A., Carlson-Kuhta, P., Zampieri, C., King, L., Chiari, L., \& Horak, F. B. (2012). ISway: a sensitive, valid and reliable measure of postural control. J Neuroeng Rehabil, 9(1), 59. doi:10.1186/1743-0003-9-59

Mancini, M., Schlueter, H., El-Gohary, M., Mattek, N., Duncan, C., Kaye, J., \& Horak, F. B. (2016). Continuous Monitoring of Turning Mobility and Its Association to Falls and Cognitive Function: A Pilot Study. The Journals of Gerontology Series A: Biological Sciences and Medical Sciences, 71(8), 1102-1108. doi:10.1093/gerona/glw019

McGinley, M., Hoffman, R. L., Russ, D. W., Thomas, J. S., \& Clark, B. C. (2010). Older adults exhibit more intracortical inhibition and less intracortical facilitation than young adults. Experimental gerontology, 45(9), 671-678. doi:https://doi.org/10.1016/j.exger.2010.04.005 
Oliviero, A., Profice, P., Tonali, P. A., Pilato, F., Saturno, E., Dileone, M., .. . Di Lazzaro, V. (2006). Effects of aging on motor cortex excitability. Neuroscience research, 55(1), 7477. doi:https://doi.org/10.1016/j.neures.2006.02.002

Papegaaij, S., Taube, W., Hogenhout, M., Baudry, S., \& Hortobágyi, T. (2014). Age-related decrease in motor cortical inhibition during standing under different sensory conditions. Frontiers in aging neuroscience, 6, 126.

Park, J. H., Mancini, M., Carlson-Kuhta, P., Nutt, J. G., \& Horak, F. B. (2016). Quantifying effects of age on balance and gait with inertial sensors in community-dwelling healthy adults. Exp Gerontol, 85, 48-58. doi:10.1016/j.exger.2016.09.018

Peinemann, A., Lehner, C., Conrad, B., \& Siebner, H. R. (2001). Age-related decrease in pairedpulse intracortical inhibition in the human primary motor cortex. Neuroscience Letters, 313(1), 33-36. doi:https://doi.org/10.1016/S0304-3940(01)02239-X

Penninx, B. W., Ferrucci, L., Leveille, S. G., Rantanen, T., Pahor, M., \& Guralnik, J. M. (2000). Lower extremity performance in nondisabled older persons as a predictor of subsequent hospitalization. The Journals of Gerontology Series A: Biological Sciences and Medical Sciences, 55(11), M691-M697.

Peterson, D. S., Plotnik, M., Hausdorff, J. M., \& Earhart, G. M. (2012). Evidence for a relationship between bilateral coordination during complex gait tasks and freezing of gait in Parkinson's disease. Parkinsonism \& related disorders, 18(9), 1022-1026. doi:10.1016/j.parkreldis.2012.05.019

Pitcher, J. B., Ogston, K. M., \& Miles, T. S. (2003). Age and sex differences in human motor cortex input-output characteristics. The Journal of physiology, 546(2), 605-613. doi:10.1113/jphysiol.2002.029454 
Plotnik, M., Bartsch, R. P., Zeev, A., Giladi, N., \& Hausdorff, J. M. (2013). Effects of walking speed on asymmetry and bilateral coordination of gait. Gait \& Posture, 38(4), 864-869.

Plotnik, M., Giladi, N., \& Hausdorff, J. (2009). Bilateral coordination of gait and Parkinson's disease: the effects of dual tasking. Journal of Neurology, Neurosurgery \& Psychiatry, $80(3), 347-350$.

Plotnik, M., Giladi, N., \& Hausdorff, J. M. (2007). A new measure for quantifying the bilateral coordination of human gait: effects of aging and Parkinson's disease. Exp Brain Res, 181(4), 561-570. doi:10.1007/s00221-007-0955-7

Seidler, R. D., Bernard, J. A., Burutolu, T. B., Fling, B. W., Gordon, M. T., Gwin, J. T., .. . Lipps, D. B. (2010). Motor control and aging: links to age-related brain structural, functional, and biochemical effects. Neurosci Biobehav Rev, 34(5), 721-733. doi:10.1016/j.neubiorev.2009.10.005

Serrien, D. J., \& Swinnen, S. P. (1997). Coordination constraints induced by effector combination under isofrequency and multifrequency conditions. Journal of Experimental Psychology: Human Perception and Performance, 23(5), 1493.

Serrien, D. J., Swinnen, S. P., \& Stelmach, G. E. (2000). Age-related deterioration of coordinated interlimb behavior. The Journals of Gerontology Series B: Psychological Sciences and Social Sciences, 55(5), P295-P303.

Shumway-Cook, A., \& Horak, F. B. (1986). Assessing the influence of sensory interaction on balance: suggestion from the field. Physical Therapy, 66(10), 1548-1550.

Sivagnanasunderam, M., Gonzalez, D. A., Bryden, P. J., Young, G., Forsyth, A., \& Roy, E. A. (2015). Handedness throughout the lifespan: cross-sectional view on sex differences as asymmetries change. Frontiers in psychology, 5, 1556. 
Smith, A. E., Ridding, M. C., Higgins, R. D., Wittert, G. A., \& Pitcher, J. B. (2011). Cutaneous afferent input does not modulate motor intracortical inhibition in ageing men. European Journal of Neuroscience, 34(9), 1461-1469. doi:10.1111/j.1460-9568.2011.07869.x

Smith, G. A., \& Brewer, N. (1995). Slowness and age: speed-accuracy mechanisms. Psychology and aging, 10(2), 238.

Sohn, Y. H., Kang, S. Y., \& Hallett, M. (2005). Corticospinal disinhibition during dual action. Experimental Brain Research, 162(1), 95-99.

Spain, R. I., St. George, R. J., Salarian, A., Mancini, M., Wagner, J. M., Horak, F. B., \& Bourdette, D. (2012). Body-worn motion sensors detect balance and gait deficits in people with multiple sclerosis who have normal walking speed. Gait \& Posture, 35(4), 573-578. doi:10.1016/j.gaitpost.2011.11.026

Springer, S., Giladi, N., Peretz, C., Yogev, G., Simon, E. S., \& Hausdorff, J. M. (2006). Dualtasking effects on gait variability: the role of aging, falls, and executive function. Mov Disord, 21(7), 950-957. doi:10.1002/mds.20848

Temprado, J.-J., Vercruysse, S., Salesse, R., \& Berton, E. (2010). A dynamic systems approach to the effects of aging on bimanual coordination. Gerontology, 56(3), 335-344.

Thigpen, M. T., Light, K. E., Creel, G. L., \& Flynn, S. M. (2000). Turning difficulty characteristics of adults aged 65 years or older. Physical Therapy, 80(12), 1174-1187.

Tombaugh, T. N., \& McIntyre, N. J. (1992). The mini-mental state examination: a comprehensive review. Journal of the American Geriatrics Society, 40(9), 922-935.

VanSant, A. F. (1988). Rising from a supine position to erect stance: description of adult movement and a developmental hypothesis. Physical Therapy, 68(2), 185-192. 
Verghese, J., Holtzer, R., Lipton, R. B., \& Wang, C. (2009). Quantitative gait markers and incident fall risk in older adults. The Journals of Gerontology: Series A, 64(8), 896-901.

Wishart, L. R., Lee, T. D., Murdoch, J. E., \& Hodges, N. J. (2000). Effects of aging on automatic and effortful processes in bimanual coordination. The Journals of Gerontology Series B: Psychological Sciences and Social Sciences, 55(2), P85-P94.

Yogev, G., Plotnik, M., Peretz, C., Giladi, N., \& Hausdorff, J. M. (2007). Gait asymmetry in patients with Parkinson's disease and elderly fallers: when does the bilateral coordination of gait require attention? Experimental Brain Research, 177(3), 336-346. doi:10.1007/s00221-006-0676-3

Zar, J. H. (1998). Biostatistical Analysis. New York, NY: Prentice-Hall.

Ziemann, U., Lönnecker, S., Steinhoff, B. J., \& Paulus, W. (1996). The effect of lorazepam on the motor cortical excitability in man. Experimental Brain Research, 109(1), 127-135. 